\title{
The Relative Influence of Place and Direction in the Morris Water Task
}

\author{
Derek A. Hamilton, Katherine G. Akers, \\ Travis E. Johnson, James P. Rice, and \\ Felicha T. Candelaria \\ University of New Mexico
}

\author{
Robert J. Sutherland \\ University of Lethbridge
}

\author{
Michael P. Weisend \\ Mental Illness and Neuroscience Discovery (MIND) Institute
}

\author{
Edward S. Redhead \\ University of Southampton
}

\begin{abstract}
Previous work from our laboratory has demonstrated that rats display a preference for directional responding over true place navigation in the Morris water task. The present study evaluated the range of situations in which this preference is observed and attempted to identify methods that favor navigation to the precise location of the escape platform in the room. A preference for directional responding over place navigation was observed in a wide range of procedures that included providing extensive training (Experiment 1), providing only platform placement experience in the absence of active swim training (Experiment 2), training navigation to multiple platform locations in a moving platform variant of the task (Experiment 3), and explicitly training navigation to a precise location in the room, versus navigation in a particular direction, regardless of the pool's position in the room (Experiments 4-5). A modest preference for navigation to the precise spatial location of the platform was observed when the pool wall was virtually eliminated as a source of control by filling it to the top with water (Experiment 6).
\end{abstract}

Keywords: spatial learning, place navigation, cognitive mapping, hippocampus, water maze

The experimental analysis of basic behavioral and learning processes involved in maze learning and spatial navigation has held a special place throughout the history of experimental psychology. Perhaps no single issue within the spatial navigation literature has been more intensely studied and debated than the question of what is learned when navigation to a particular place is reinforced. Tolman, Ritchie, and Kalish (1946) contrasted place and response learning in the simple T-maze by training animals to either perform a particular response (e.g., always enter the arm to the right) or to navigate to a specific spatial location in the room regardless of the particular arm where reinforcement occurred. The ability of rats to learn where to navigate independently of specific motor responses represents what Tolman et al. (1946) termed a place disposition, a concept that became a central feature of Tolman's cognitive mapping theory (Tolman, 1948) and subsequently the influential mapping theory of O'Keefe and Nadel

Derek A. Hamilton, Departments of Psychology and Neurosciences, and Katherine G. Akers, Travis E. Johnson, James P. Rice, and Felicha T. Candelaria, Department of Psychology, University of Mexico, Albuquerque; Robert J. Sutherland, Canadian Centre for Behavioural Neuroscience, University of Lethbridge, Alberta Canada; Michael P. Weisend, Department of Psychology, Mental Illness and Neuroscience Discovery (MIND) Institute, Albuquerque, New Mexico; Edward S. Redhead, School of Psychology, University of Southampton Southampton, United Kingdom.

The authors thank Isaac Franco for assistance with data collection and Jeani Sarosy for assistance with preparation of the manuscript.

Correspondence concerning this article should be addressed to Derek A. Hamilton, MSC03 2220, Department of Psychology, University of New Mexico, Albuquerque, NM 87131. E-mail: dahamilt@unm.edu
(1978). During the early years of this debate place learning and the place disposition were contrasted not only with the response disposition, but several alternative behavioral processes including approach/avoidance tendencies (Hull, 1943, 1934a, 1934b), simple and complex guidance (Deutsch, 1960), and directional responding (Blodgett, McCutchan, \& Mathews, 1949).

Blodgett et al. (1949) noted that the apparent place disposition demonstrated by Tolman et al. (1946) might reflect learning to move in the direction of reinforcement within the room and maze rather than true place navigation. To contrast the relative influence of response, place, and directional strategies, Blodgett et al. (1949) rotated and/or repositioned a T-maze from trial to trial and systematically manipulated the reinforced arm such that only a single form of responding would reliably result in reinforcement. For the response only groups (Groups III, VIII, and IX), reinforcement was always located in one arm of the maze (left or right), and because the maze was repositioned and rotated for each trial, the place and direction of reinforcement varied such that the particular turning response was the only response that reliably resulted in reinforcement. For the direction only groups (Groups II and XI), reinforcement was always located in the same direction in the room and maze (e.g., to the east). The reinforced arm (left or right) and the precise location of reinforcement in the room varied from trial to trial, making the directional response the only response that always resulted in reinforcement. Finally, for the place only group (Group I), reinforcement was always located the same place in the room regardless of the apparatus position. The reinforced arm (left or right) and the direction of reinforcement in the room and maze varied from trial to trial, thus, navigating to the same spatial location was the only response that always resulted in reinforce- 
ment. The groups for which a single directional or turning response reliably resulted in reinforcement made the fewest errors, whereas the place only group committed the most errors. Based on this outcome Blodgett et al. (1949) concluded that the relative contribution of place information to performance in the T-maze is negligible in comparison to response and directional information, thus, the apparent place disposition reported by Tolman et al. (1946) when the maze remained stationary can reasonably be explained in terms of directional responding rather than true place navigation. Similar observations have been reported in other dryland maze tasks (Skinner et al., 2003; Stringer, Martin, \& Skinner, 2005), and strong support for the idea that animals learn a directional response more readily than navigation to precise spatial locations in the Morris water task has also been demonstrated (Hamilton et al., 2007; Weisend et al., 1995).

Over the past three decades, the Morris water task (Morris, 1981, 1984; Sutherland \& Dyck, 1984) has become a model behavioral task for the measurement of place navigation and learning. In this task, rats are trained to navigate to a hidden escape platform in a circular pool of cool, opaque water. The platform remains in the same spatial location and multiple release locations are used, thus, simple motor responses are ineffective and it is generally agreed that animals learn to navigate to the platform based upon its fixed spatial relationship to the available distal visual cues. The fact that the platform is in a fixed spatial relationship to distal visual stimuli, however, is neither sufficient to conclude that animals learn to navigate to a precise location in the water task, nor does it rule out the possibility that animals learn to move in the direction of the platform within the room and pool.

To contrast directional responding and place navigation in the Morris water task, Hamilton et al. (2007) trained rats to swim to a hidden escape platform and then administered a single no-platform probe trial with the pool repositioned in the room such that the absolute spatial location of the platform in the room was centered in the opposite quadrant of the pool. For example, if an animal was trained with the pool in position 1 (see Figure 1) and the platform in location B, moving the pool to position 2 for the no-platform probe trial could result in navigation to the absolute location of the platform (location B) or navigation in the direction of the platform in the room and apparatus (to the east), which would result in navigation to the relative location of the platform in the pool (location C). The results clearly showed that rats swam in the direction of the platform and persisted in searching at the relative location rather than the absolute place where the platform was located. Furthermore, animals treated the relative location as if it were the absolute (trained) location, while treating the absolute location as if it were an arbitrarily selected, untrained location. None of the procedural variants or experimental manipulations utilized by Hamilton et al. (2007) altered this basic pattern of observations, including the number of training trials, the amount of time animals remained on the escape platform during each trial, or the presence of other competing sources of control. Thus, the results of Hamilton et al. (2007) suggest that directional responding may represent a more general process than true place navigation by which animals navigate to the escape platform in the water task.

The present study has two broad and closely related goals that are aimed at (1) evaluating the range of water task training procedures and methods for which directional responding is observed,
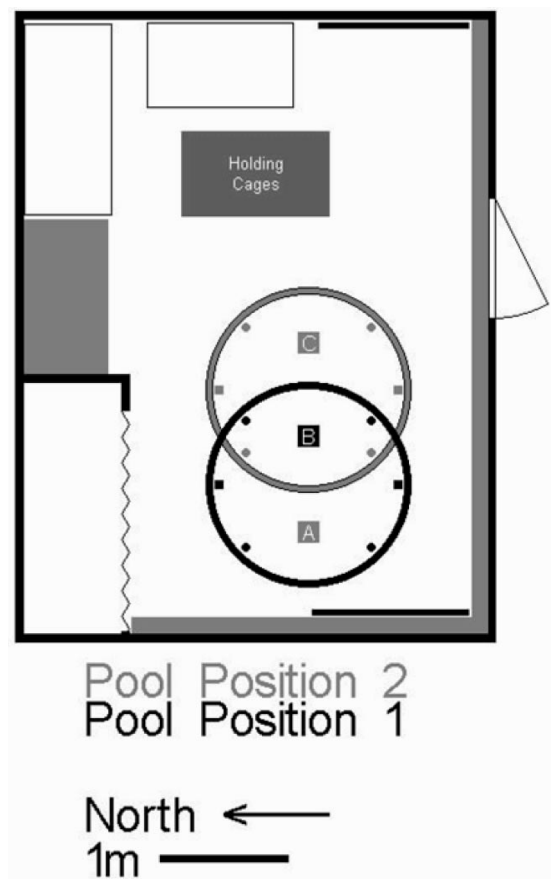

Figure 1. Layout of the room used for Experiments 1, 2, 3, and 6 showing the two locations where the pool could be positioned in the room. Pool positions 1 (black) and 2 (gray) represent the same pool positions used by Hamilton et al. (2007; see their Figure 1). Each pool position was separated by $75 \mathrm{~cm}$ (the radius of the pool). The platform was typically located at the black rectangle labeled $\mathrm{B}$; the gray rectangles (locations A and $\mathrm{C}$ ) mark comparison locations (relative/opposite) used for probe trial analyses. The small circles (SW, SE, NW, NE) represent release points used during training trials and the rectangles (north-most and south-most points) represent release points used during no-platform probe trials; Black indicates release points for pool position 1 and gray indicates release points used for pool position 2. Prominent distal visual cues (e.g., a chalkboard on the west wall) are marked by black or gray rectangles. There were two doors in the room (one of which was covered by a tarp, shown on the east wall) and the other (south wall), which was always closed during testing. There were no windows in the room; The curtain shown on the north wall covered a small storage area and was always closed. The room was approximately $3.5 \mathrm{~m}$ in height and most of the distal stimuli shown here extended at least $1.25 \mathrm{~m}$ above the top of the pool. The long gray rectangles mark the location of wooden wiring channels that were located near the top of the room.

and (2) attempting to identify procedures that effectively support navigation to the absolute platform location over directional responding. As such, the experiments comprising the present study share the goal of evaluating the relative influence of place and direction information in the water task, as well as addressing the generality of directional responding in the water task. A series of six experiments is reported in which several standard and some novel variants of the water task and its associated training procedures were employed. Whether animals perform directional responses or navigate to the absolute spatial location of the platform during a critical test trial with the pool repositioned is evaluated in each of the experiments. Experiment 1 addressed the relationship between the overall amount of training and whether animals perform a directional response, and further provides data to complement the findings of Hamilton et al. (2007) relevant to this issue. 
Experiments 2-6 evaluated the effects of various forms of training on directional responding and place navigation, including whether test performance is differentially affected by platform placement training, moving platform training, training in which the pool is repositioned for each training trial but the escape platform remains in the same absolute or relative location in the pool, and training where control by the apparatus (pool wall) is reduced. After outlining the methods common to each of the experiments, the rationale, specific methods, and results for each experiment are presented in turn.

\section{General Method}

\section{Subjects}

Subjects were male hooded Long-Evans rats (Charles River Laboratories, Wilmington, MA or Harlan, Indianapolis, IN) that were approximately 90 days of age at the beginning of the experiments. All animals were pair-housed in plastic cages on a $12 \mathrm{~h}$ light:dark cycle with food and water available ad libitum. Behavioral testing was performed during the light phase. All procedures for the studies reported here were approved by the Institutional Animal Care and Use Committee (IACUC) at the University of New Mexico.

\section{Apparatus}

The circular pool (1.5 m diameter, $48 \mathrm{~cm}$ high) was painted white on the inner surface and was placed on a wooden frame (48 $\mathrm{cm}$ tall) that rested on appliance rollers, making it possible to easily move the pool when it was filled with water. The escape platform was constructed of plastic with a $16 \mathrm{~cm} \times 16 \mathrm{~cm}$ top surface and a height of $25 \mathrm{~cm}$. The pool was filled to a depth of 26 $\mathrm{cm}$ with cool water $\left(22{ }^{\circ} \mathrm{C}\right)$ that was made opaque by adding a small amount $(\sim 2$ oz. $)$ of powdered white tempura paint. The testing room contained two doors (one of which was covered by a tarp) and numerous other distal visual cues (e.g., posters, a chalk board) and the room walls formed a complex geometry (see Figure 1). There were no windows in the room and the doors were always closed during testing. The top of the pool was approximately 75 $\mathrm{cm}$ from the floor and the room walls were approximately $3.5 \mathrm{~m}$ tall. Many of the distal visual stimuli were placed high on the walls so that they were not entirely obscured by the pool wall. Behavior was videotaped via an overhead camera and digital camcorder. The digital video was transferred to a Linux workstation for tracking and analysis.

\section{Procedure, Dependent Measures, and Analyses}

Unless otherwise noted, the training and testing procedures and parameters were as follows. For hidden platform training trials rats were released facing the wall of the pool and were retrieved from the platform after 5-10 s. Latency to navigate to the platform served as the primary training dependent measure for all experiments. If a rat did not find the platform within $60 \mathrm{~s}$ it was retrieved and placed on the platform for 5-10 s. Animals were placed in a holding cage for approximately $5 \mathrm{~min}$ between trials. For noplatform probe trials animals were released from one of two points (north or south) selected at random with the constraint that each release point was used equally often within a given experimental condition. Probe trials lasted for $30 \mathrm{sec}$. Four dependent measures were taken during the probe trials for each of two critical locations in the pool that were the same size as the platform surface. One critical location was the absolute location of the platform in the room and the other was in the diametrically opposite quadrant. When the pool is repositioned, the opposite location corresponds to the relative location of the platform in the pool during training, and is the location to which a directional response would be expected. If the pool is not repositioned, the opposite location serves as a comparison location that has the same spatial relationship to the absolute location, as does the relative location for conditions in which the pool is repositioned (i.e., the relative and absolute locations are in opposite quadrants when the pool is repositioned). For example, if the pool was in position 1 and the platform was in location B (see Figure 1) during training, and the pool remained in position 1 for the probe trial, the opposite location would correspond to location A. If the pool was repositioned (to position 2) for the probe then location $\mathrm{B}$ corresponds to the absolute location and location $\mathrm{C}$ corresponds to the relative/opposite location. The number of times each critical location was crossed and the average distance from each location during the probe trial were measured. The latter measure was adapted from the goal proximity measure described by Gallagher, Burwell, and Burchinal (1993). The latency to enter and the amount of time spent in a circular region (66 $\mathrm{cm}$ in diameter) centered on each of the critical locations were also measured. Unless otherwise noted, all statistical tests reported for probe trials include one between-subjects factor (group) and one within-subject factor (location). For all experiments reported here, one critical location was the absolute location of the platform in the room. The other critical location was usually the opposite/ relative location, however, comparison locations for Experiments 4 and 5 were slightly modified and are described in the appropriate Method sections. All statistical tests are significant at $p<.05$ unless otherwise noted.

\section{Experiment 1}

Because several studies have claimed that rats navigate to places early in training and switch to a response-based strategy later in training (Chang \& Gold, 2003; Packard \& McGaugh, 1996). Hamilton et al. (2007) evaluated whether rats navigate to the absolute location of the platform (early) and then perform directional responding (later) in the Morris water task. Rats were given 8 training trials, which is sufficient for rats to begin navigating directly to the platform, or 36 trials, which extends well beyond the point at which animals reach asymptotic levels of performance (8-12 trials). When the pool was repositioned for a probe trial at the end of training, rats in both conditions performed a directional response and in many respects treated the absolute location as if it were an untrained location. This observation is inconsistent with the idea that animals switch from place-responding to directionalresponding within this range of trials. Further, the claim that animals shift from place navigation to another form of responding is clearly at odds with the results of other studies showing that rats learn turning responses and directional responses rather easily but learn to navigate to places with more difficulty (Blodgett et al., 1949; Skinner et al., 2003). Because navigation to places requires more training (Blodgett et al., 1949; Skinner et al., 2003; Stringer et al., 2005), perhaps providing more extensive training in the 
water task would shift the response bias of rats from directional to place navigation, a possibility that was not investigated by Hamilton et al. (2007). To evaluate this possibility, rats were given extensive hidden-platform training (240 trials) and tested for directional responding versus navigation to the absolute platform location at 4 separate points during training.

\section{Method}

Subjects. Subjects were 16 rats that were randomly assigned in equal numbers $(n=8)$ to one of two experimental conditions (No Shift or Shift).

Design and procedure. Twelve hidden-platform training trials (3 blocks of 4 trials) were conducted during each day of training. During each block rats were released once from each of four release points (NW, SW, NE, SE; see Figure 1). The order of release points was selected randomly without replacement. During training the pool was positioned at location 1 or 2 , with an equal number of rats from each condition trained with each pool position. The platform was placed at location B throughout training regardless of the pool position. A total of 20 days of training were conducted; 5 days of successive training were conducted with two days of no-training in between. A single no-platform probe trial was conducted at the end of every fifth day of training for a total of 4 probe trials (i.e., after 60, 120, 180, and 240 trials). During the probe trial the pool either remained in the same position used during training (No Shift group) or was repositioned by $75 \mathrm{~cm}$ to the position that was not used during training (Shift group).

\section{Results}

Hidden platform training. To simplify presentation of the training results, only data from the first five days and the final trial block (on Day 20) were analyzed. Latency data were averaged for each of the training trial blocks for each of the first five days and analyzed with a repeated measures ANOVA with trial block (115) and group (Shift or No Shift) as factors. There was a main effect of trial block, $F(14,196)=54.69$, which resulted from a decrease in escape latency across trial blocks, $M(S D)_{\mathrm{Block} 1}=$ $30.38 \mathrm{~s}$ (9.72); $M(S D)_{\text {Block15 }}=3.47 \mathrm{~s}(1.32)$. The main group effect and the Trial Block $\times$ Group interaction were not significant, both $p \mathrm{~s}>.44$. The group difference for the final trial block of training on Day 20 was also not significant, $F(1,14)<1$; $M(S D)_{\text {NoShift }}=2.41 \mathrm{~s}(0.55) ; M(S D)_{\text {Shift }}=2.44 \mathrm{~s}(0.22)$.

No-platform probe trials. During each of the four probe trials, all 8 rats in the no-shift group navigated to the absolute region first, whereas all 8 rats in the Shift group navigated to the relative region first. Representative probe trial swim paths for each group are shown in Figure 2 and group means for each of the probe trial dependent measures for the first (Day 5) and last (Day 20) probe trials are shown in Figure 3 (Data for the second (Day 10) and third (Day 15) probe trials are not shown although they were comparable to the values represented in Figure 3 and were analyzed and reported below).

Separate analyses of variance (ANOVAs) were conducted for each of the four probe trials with group (No Shift or Shift) as a between-subjects factor and location (absolute vs. opposite/ relative) as a within-subject factor, where the opposite location corresponds to the relative location for the Shift group. For all four

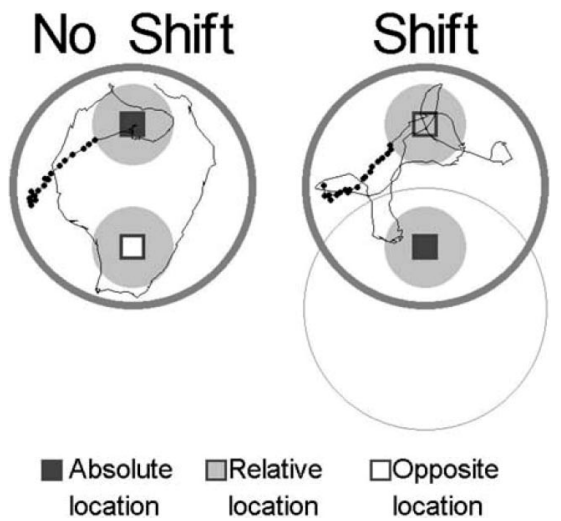

Figure 2. Representative probe trial swim paths for animals from the Shift and No Shift groups of Experiment 1. Paths were selected for animals with median latencies to enter the preferred region during the probe trial (absolute for the No Shift group and relative for the Shift group). The large, thin circle shown for the Shift animal indicates the pool position used during training. The thick circles indicate the pool position during the probe trial. The light gray circles within the pool mark the two critical regions (66 cm diameter) around the absolute (dark gray square), relative (light gray square) or opposite location (open square) used for analysis. The initial trajectory, defined as the path taken from the release point until one of the two critical circular regions was entered, is shown in filled black circles. The remainder of the path for the probe trial is shown as a thin black line.

probe trials the group and location main effects were not significant for latency to enter the critical regions, all $p s>.13$, or for average distance from the critical locations, all $p \mathrm{~s}>.24$. A significant group main effect was observed for time in region on the Day 10 probe trial (No Shift $>$ Shift), $F(1,14)=5.17$, indicating that the No Shift group spent more time in the two critical regions than the Shift group. The group main effect failed to reach significance for this measure on the other probe days, all $p s>$.068. Significant location main effects were observed for time in region and number of location crossings on the Day 5 and 10 probe trials (absolute $>$ opposite), all $p$ s $<.03$, indicating that animals spent more time in the absolute location and crossed the absolute location more frequently than the opposite location. The location main effects were not significant for the Day 15 and 20 probe trials, all $p s>.062$, however, there was a numerical difference favoring the absolute location. The Group $\times$ Location interaction was significant for every probe trial for all four dependent measures; Day 5, all $p$ s $<.001$; Day 10, all $p \mathrm{~s}<.001$; Day 15, all $p \mathrm{~s}<.015$; and Day 20, all $p \mathrm{~s}<.006$.

Follow-up comparisons of measures for the two critical locations within each group revealed that, for each probe trial, No Shift animals entered the absolute region faster than the opposite region, all $p \mathrm{~s}<.001$; spent more time in the absolute region than the opposite region, all $p \mathrm{~s}<.02$; crossed the absolute location more frequently than the opposite location, all $p \mathrm{~s}<.034$; and navigated closer to the absolute location than the opposite location, all $p \mathrm{~s}<$ .032. Shift animals entered the relative region faster than the absolute region during all four probe trials, all $p$ s $<.022$, and spent more time in the relative region during all four probe trials, all $p s<.032$. Shift animals navigated significantly closer to the relative location during the Day $5-15$ probe trials, all $p \mathrm{~s}<.006$, 


\section{Day 5}
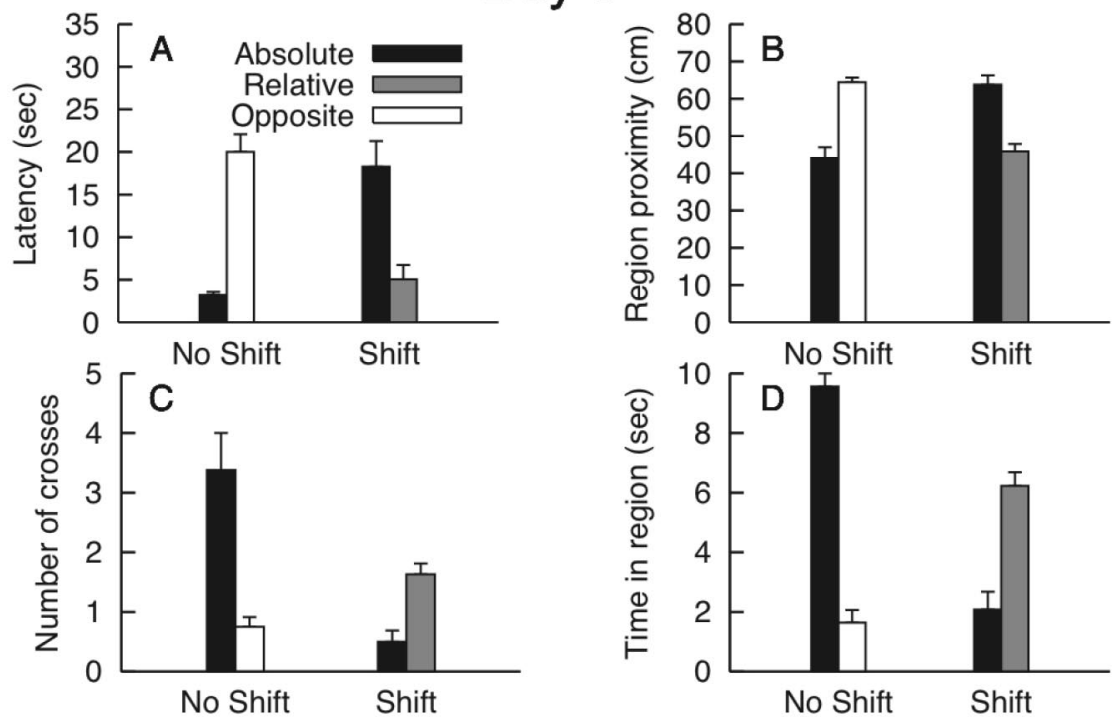

Day 20
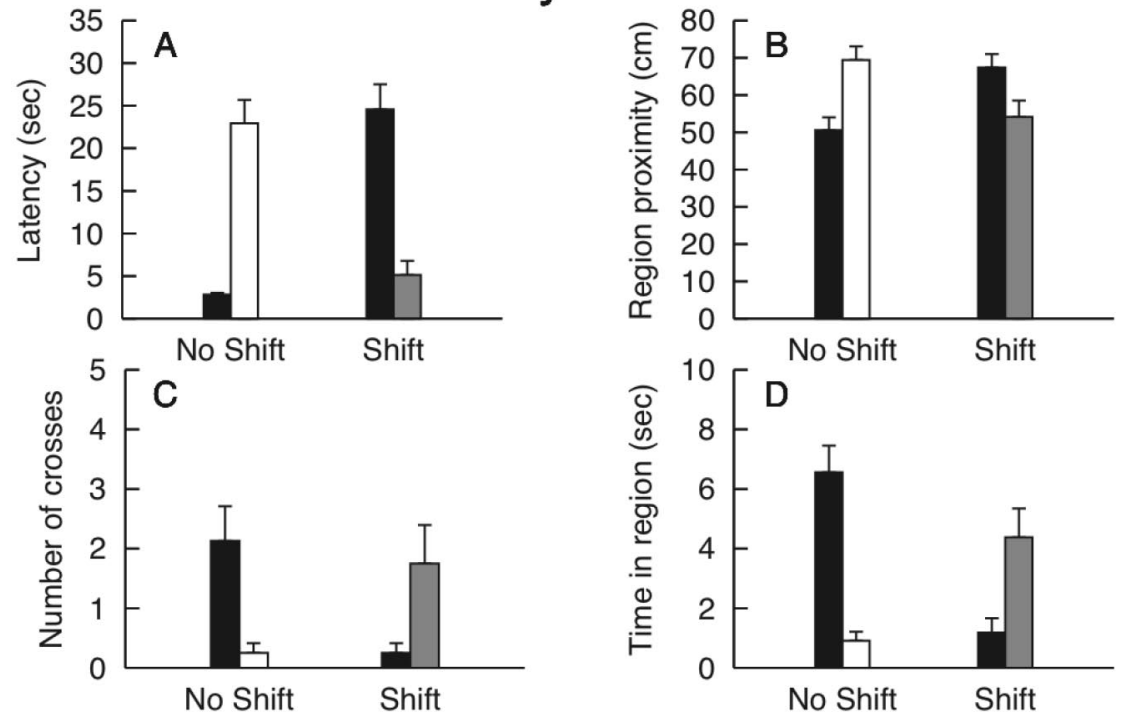

Figure 3. Probe trial dependent measures (Mean + SEM) for the No Shift and Shift groups of Experiment 1. Data from the Day 5 and Day 20 probe trials are shown. A: Latency to enter the $66 \mathrm{~cm}$ diameter circular region around the two locations of interest. B: Average distance from the two critical locations. C: Number of times each critical location was crossed. D: Time spent in each of the two critical circular regions.

but the location effect was not significant for the Day 20 probe trial, $p=.11$, even though the means were comparable to those of the previous probe trials. For all four probe trials Shift animals crossed the relative location more frequently, however the location effect was only significant on Day $5, F(1,7)=21.59$. Although the Location effect was not significant for number of crosses for the probe trials of Days 10-20, all $p s>.071$, the number of times the relative location was crossed was significantly greater than zero for these three probe trials, all $p s>.032$, whereas the number of times the absolute location was crossed was only significantly greater than zero on the Day 10 probe, $p=.033$, all other $p$ s $>.08$. Thus, overall the significant two-way interactions described above and illustrated in Figure 3 can be attributed to the fact that the No Shift animals displayed a preference for the absolute location whereas Shift animals displayed a preference for the relative location.

Comparisons of performance measures for the relative location for the Shift group and measures for the absolute location for the No Shift group were conducted to evaluate group differences for the "preferred" locations. Rats in the No Shift group navigated to the absolute region faster than rats in the Shift group navigated to 
the relative region on the Day 10 probe trial, $F(1,14)=5.76$, however, group differences for latency were not detected during any of the other probe trials, all $p s>.083$. No Shift rats spent more time in the absolute region than Shift rats spent in the relative region on the Day 5 and 10 probe trials, both $p s<.023$, however, significant group differences were not detected for the probe trials on Days 15 and 20, both $p s>.11$. Similarly, No Shift rats crossed the absolute location more frequently than Shift rats crossed the relative location on the Day 5 and 10 probe trials, both $p$ s $<.02$, but no significant differences were detected on the Day 15 and Day 20 probe trials for this measure, both $p$ s $>.075$. No significant Group differences were observed for average proximity, all $p s>.34$.

Comparisons of measures for the relative location in the Shift condition with measures for the opposite location for the No Shift condition revealed significant differences for all dependent measures for all four probe trials, all $p \mathrm{~s}<.04$, with the exception that there was no main effect for number of times the critical location was crossed for the Day 15 probe trial, $p=.15$. Shift rats navigated to the relative region faster than No Shift rats navigated to the opposite region, spent more time in the relative region than rats in the No Shift condition spent in the opposite region, crossed the relative location more frequently than No Shift animals crossed the opposite location, and navigated closer to the relative location than rats in the No Shift group navigated to the opposite location.

Comparisons of measures for the absolute location in the Shift condition and the opposite location in the No Shift group were conducted to evaluate group differences for the "nonpreferred" location. These comparisons revealed no significant group differences for any dependent measure during any of the probe trials, all $p$ s $>.20$.

\section{Discussion}

The results of Experiment 1 demonstrate that rats display a preference for directional responding over navigation to the absolute spatial location of the escape platform in the Morris water task even after extensive training. Rats in the Shift and No Shift groups learned to navigate to the escape platform at comparable rates and to nearly identical levels of performance. When the pool was repositioned for the probe trials, Shift animals showed a clear preference for the relative location compared to the absolute location, whereas No Shift rats showed a clear preference for the absolute location. Although No Shift rats showed superior levels of performance for the absolute location compared to the relative location for the Shift rats, these differences were not consistent across measures or probe trials. With respect to overall patterns of performance, Shift rats treated the relative location in much the same way that No Shift rats treated the absolute location. Significant differences between measures for the opposite location for the No Shift group and the relative location for the Shift group further support the conclusion that the relative location was treated as though Shift animals were trained to navigate to this location in the room even though they were never explicitly trained to do so. No differences were detected in how the Shift rats treated the absolute location and how No Shift rats treated an untrained location in the quadrant opposite the platform quadrant, supporting the conclusion that Shift animals treated the absolute location as if it were an untrained location.
The present findings indicate that a preference for navigation to the absolute spatial location of the platform (over directional responding) does not develop with extensive training. Together with the results of Hamilton et al. (2007), the present findings suggest that directional responding is not a transient phenomenon. Of course, it is possible that a preference for navigating to absolute places might be observed with even more extensive training than was provided here, however, at 240 trials the present study used roughly 6 times more training trials than is typically used in the water task. Thus, a more conservative conclusion is that directional responding occurs over a wide range of training trials, including the typical number of training trials used in the water task.

\section{Experiment 2}

Given that the extent of active swim training does not affect whether rats perform a directional response or navigate to the absolute platform location, Experiment 2 was undertaken to determine whether a different form of experience might favor navigation to places over directional responding. Several studies have presented positive evidence that place navigation in the water task can be facilitated based upon prior experience viewing the distal cues from the platform location without active swim training in the test environment (Devan et al., 2002; Keith \& McVety, 1988; Pearce, Roberts, Redhead, \& Prados, 2000; Sutherland \& Linggard, 1982). Whether such experience results in learning the precise location of the platform remains a matter of debate (Sutherland, Chew, Baker, \& Linggard, 1987; Sutherland \& Hamilton, 2004; Chew, Sutherland, \& Whishaw, 1989; Hamilton et al., 2007), however, if platform experience favors learning to navigate to the absolute platform location then the probe trial methods used in the previous experiment should be capable of revealing such a preference. Hamilton et al. (2007) allowed rats to remain on the platform for 5-30 s at the end of each trial and found that directional responses were performed regardless of the amount of time on the platform, however, the Hamilton et al. (2007) study did not evaluate the effects of platform experience in the complete absence of active swim training. In Experiment 2 rats were trained to navigate to a hidden escape platform in a separate room used only for pretraining. After pretraining, animals were taken to the test room and placed on the platform for $2 \min$ (for 1 or 4 trials), after which the pool was repositioned or remained in the same location and a no-platform probe trial was conducted. If rats learn the precise location of the platform then a preference for the absolute location should be observed. Alternatively, if animals learn the general direction of the platform within the apparatus and room reference frames then a preference for the relative location should be observed.

\section{Method}

Subjects. Subjects were 40 experimentally naive rats that were randomly assigned to one of five conditions.

Apparatus. The testing room and pool used in Experiment 1 were used for the no platform probe trial of Experiment 2. A different room was used for the first phase of Experiment 2 (referred to as room 2 throughout the remainder of the paper; the room depicted in Figure 1 is referred to as room 1). Room 2 was comparable to room 1, although room 2 was slightly larger and contained different distal visual cues. 
Design and procedure. Experiment 2 was conducted in three phases. The purpose of phase 1 was to provide animals with training in the water task prior to platform placement experience. During phase 1 all rats were given 16 hidden-platform training trials in a different room (room 2) from that used for the subsequent phases. All trials for phase 1 were given during a single training session with the platform in a fixed location centered in one quadrant. During each block of 4 trials animals were released from each of four release points in a pseudorandom sequence. The number of training trials was selected to ensure that all animals learned the hidden platform task and to reduce the likelihood that animals would jump from the platform during platform placement experience. At the end of phase 1 the pool was drained and transported to room 1 for phases 2 and 3. This resulted in a delay of approximately $60 \mathrm{~min}$ between the end of phase 1 and the beginning of phase 2. During phase 2 a total of 32 animals were given platform placement experience and 8 received no experience (No Placement group). For half the rats that received placement experience the pool was in position 1 and for the other half the pool was in position 2. The platform was in location B for all rats. To determine whether the amount of placement experience influenced performance, half of the placement rats received 1 placement trial and the other half received 4 placement trials. Rats were placed on the platform for 2 min after which they were removed and returned to their home cage for an intertrial interval of $2 \mathrm{~min}$. This allowed sufficient time for the pool to be repositioned and the water to settle prior to the no-platform probe trial of phase 3 . There were no instances where rats jumped from the platform during phase 2 and, therefore, no animals inadvertently received active swim training. Half of the animals that received placement experience during phase 2 were assigned to a Shift condition $(n=16$; 4 from each combination of pool position and number of placement trials during phase 2) and the other half were assigned to a No Shift group $(n=16$; 4 from each combination of pool position and number of placement trials during phase 2). For Shift animals the pool was repositioned for the phase 3 probe trial as described in Experiment 1. The platform was removed and immediately following the 2 min delay animals were released from a location equidistant from the two critical regions (the north-most or southmost points shown in Figure 1) and allowed to swim for $30 \mathrm{~s}$.

\section{Results}

Hidden platform training. Latency data were averaged for each of the 4 training trial blocks and analyzed with a repeated measures ANOVA with trial block (1-4), and group (No Placement, No Shift: 1 or 4 placement trials, and Shift: 1 or 4 placement trials) as factors. There was a main effect of trial block, $F(3$, $105)=61.40$, resulting from a decrease in latency across the trial blocks. The main effect of group was not significant, $F(4,35)=$ $1.21, p=.32$, nor was the interaction, $F(12,105)=1.76, p=$ .064. Mean group latencies during the final trial block ranged from $3.75 \mathrm{~s}$ (No Shift-1 placement trial) to $5.88 \mathrm{~s}$ (No Placement).

No-platform probe trial. Group (Shift vs. No Shift), number of trials, and location (absolute vs. opposite/relative) were included as factors. Rats given four placement trials entered the regions of interest faster than rats given one trial, $F(1,28)=5.57$, and navigated closer to the critical analysis locations than rats given 1 placement trial, $F(1,28)=5.84$. The number of trials did not have a significant effect on latency to enter the critical regions or the number of times the critical locations were crossed, both $p \mathrm{~s}>.17$. Inspection of the swim paths and quantitative data indicated that the number of placement trials did not alter the pattern of location effects for the two groups. Consistent with this observation, the number of placement trials was not involved in any significant interactions with the group and location factors, all $p \mathrm{~s}>.10$. Therefore, to simplify presentation of the results and increase statistical power, the remainder of the analyses was conducted excluding number of trials as a factor.

Representative probe trial swim paths for rats from the No Placement, No Shift, and Shift groups of Experiment 2 are shown in Figure 4 and group means for each of the probe trial dependent measures are shown in Figure 5. In the No Placement group, 4 animals entered the absolute region first (location B) and the other 4 animals entered the opposite region first (location A or location C). In the No Shift condition, 11 of the 16 rats (five given 1 trial and six given 4 trials) navigated first to the absolute region of the pool where the platform was located during platform placement. Of the 16 rats in the Shift group, 12 navigated to the relative region first (five given 1 trial and seven given 4 trials).

To simplify presentation of the results, we report separate location analyses for each group and separate group analyses for each location. Rats in the No Shift group entered the absolute region significantly faster than the opposite region, $F(1,15)=11.86$; There were numerical differences in favor of the absolute location for the other three dependent measures, however, none of these were significant, all $p \mathrm{~s}>.06$. There were numerical differences in favor of the relative location for the Shift group, however, there were no significant location effects for any of the four dependent measures, all $p s>$.20. There were no remarkable and consistent numerical differences for the critical regions in the No Placement group and none of the location effects were significant, all $p \mathrm{~s}>$ .19. There were significant group effects for all four dependent measures taken for the absolute location, all $p \mathrm{~s}<.03$. Post hoc analyses with Fisher's $L S D$ revealed that the No Shift group spent significantly more time in the absolute region than both the Shift

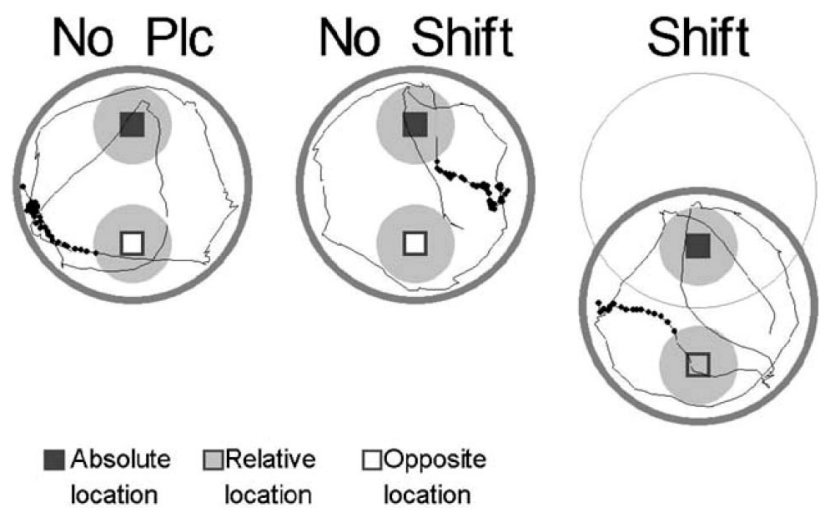

Figure 4. Representative probe trial swim paths for animals from the No Placement, Shift and No Shift groups of Experiment 2. Paths were selected for animals with median latencies to enter the preferred region during the probe trial (absolute for the No Shift group and relative for the Shift group; median latency to enter either region was used for the No Placement group). Conventions for representing locations, critical regions, pool positions, and swim paths are the same as those described for Figure 2. 

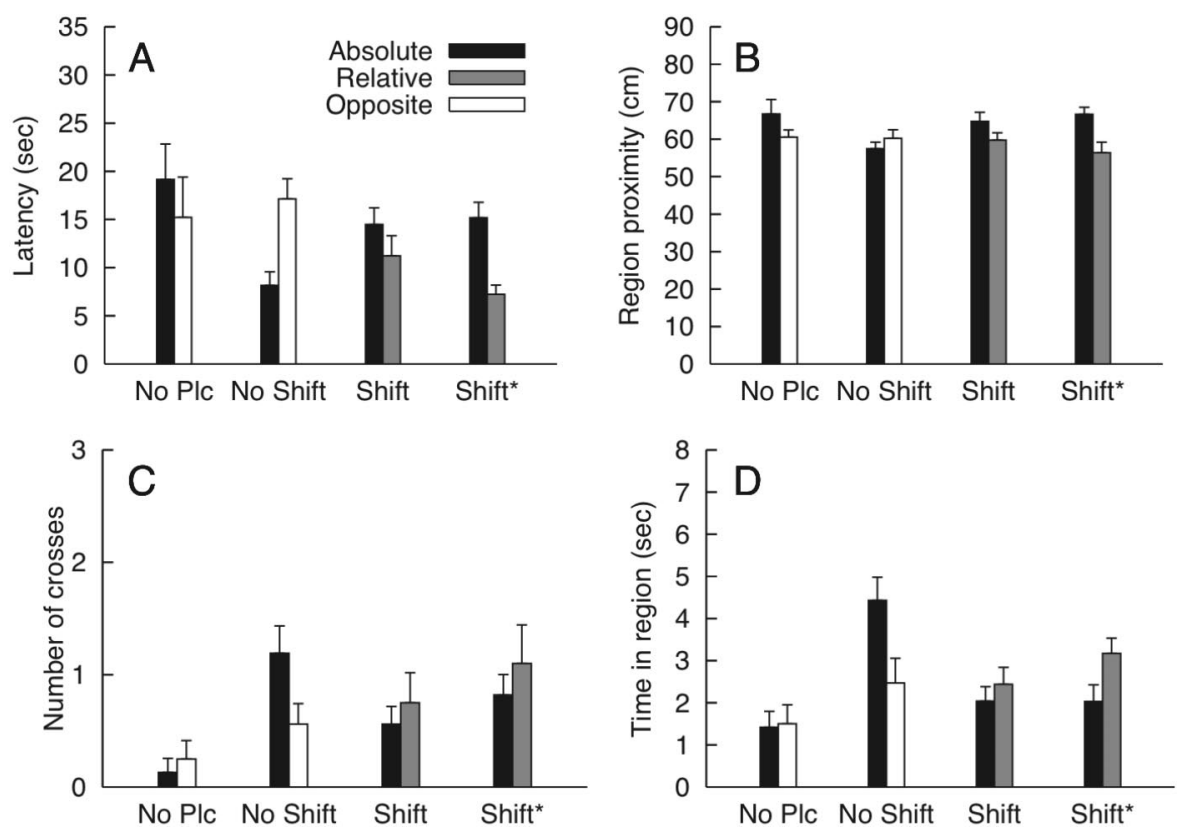

Figure 5. Probe trial dependent measures (Mean + SEM) for the No Placement (No Plc), No Shift, and Shift groups of Experiment 2. Shift* represents mean performance for the subset of Shift animals that displayed a clear preference for one location $(n=5$; Three animals that did not show a preference for either location were excluded from Shift*). A: Latency to enter the $66 \mathrm{~cm}$ diameter circular region around the two locations of interest. B: Average distance from the two critical locations. C: Number of times each critical location was crossed. D: Time spent in each of the two critical circular regions.

and No Placement groups, both $p \mathrm{~s}>.003$, entered the absolute region more quickly than the Shift and No Placement groups, both $p s<.02$, crossed the absolute location more frequently than the Shift and No Placement groups, both $p s<.03$, and navigated closer to the absolute location than the Shift and No Placement groups, both $p$ s $<.028$. The Shift and No Placement groups did not significantly differ for any of the four dependent measures for the absolute location, all $p \mathrm{~s}>.14$. There were no significant group differences for any measures for the opposite/relative location, all $p s>.19$.

In the interest of more thoroughly evaluating whether there was any evidence for a location preference in the Shift and No Shift groups, we inspected the individual swim paths for all animals in both groups. Based on the amount of time spent in each critical region and the number of location crosses, 11 of the 16 No Shift rats showed a clear preference for the absolute location and the other five showed a modest preference for the opposite region. In contrast, 11 of the 16 Shift rats showed a clear preference for the relative location while the remaining five showed no clear preference for either location. All 16 No Shift rats crossed one of the critical analysis locations at least once, whereas only the 11 Shift rats that showed a preference for the relative location actually crossed one of the critical locations at least one time. The remaining five Shift rats tended to circle around the edge of the pool and took much longer than the other rats to enter either of the critical analysis regions; $M_{\text {absolute }}=12.93 \mathrm{~s}, S D=10.2 ; M_{\text {relative }}=$ $19.97 \mathrm{~s}, S D=10.09$. To evaluate whether there was a difference in location preference between the groups we conducted an additional set of analyses which only included those rats that crossed one location at least one time; No Shift $(n=16)$, Shift $(n=11)$. Means for the 11 Shift animals are labeled "Shift*" in Figure 5. The main effects of location were not significant for any of the dependent measures; all $p \mathrm{~s}>.18$. There was a significant group effect for time in the critical analysis regions (No Shift $>$ Shift), $F(1,25)=4.59$, however, none of the remaining group effects were significant, all $p \mathrm{~s}>$.10. There were significant Location $\times$ Group interactions for latency, time in region, and proximity (all $p \mathrm{~s}<.025)$. The Location $\times$ Group interaction for number of crosses was not significant, $p=.13$. Location differences for the No Shift group were already reported above. In the Shift* group there was a significant location effect for latency; relative < absolute, $F(1,10)=22.36$; and proximity; relative $<$ absolute, $F(1,10)=6.62$. The Shift* group spent more time in the relative region, however, this difference was not significant, $F(1,10)=$ $4.31, p=.065$. The location effect for number of crosses was not significant, $p=.56$. The No Shift group spent more time in the absolute region than the Shift* group, $F(1,25)=10.50$, had lower latencies to enter the absolute region than the Shift* group, $F(1$, $25)=10.47$, and navigated closer to the absolute location than the Shift* group, $F(1,25)=8.55$. The group effect for the number of times the absolute location was crossed was not significant, all $p \mathrm{~s}=.28$, although it should be noted that the No Shift group crossed the absolute location more frequently than the Shift* group. Comparisons of the opposite location measures for the No Shift group and the relative location measures for the Shift* group revealed that the Shift* group entered the relative region faster than the No Shift group entered the opposite region, $F(1,25)=$ 13.87. The Shift* group also spent more time in the relative region 
than No Shift rats spent in the opposite region, crossed the relative location more frequently than No Shift rats crossed the opposite location, and navigated closer to the relative location than No Shift rats navigated to the opposite location, however, none of these differences were significant, all $p \mathrm{~s}>.15$. Comparisons of the absolute location measures for the No Shift group and the relative location measures for the Shift* group failed to detect significant group differences for any of the dependent measures, all $p \mathrm{~s}>.09$. Comparisons of the absolute location for the Shift* group and the opposite location for the No Shift group also failed to detect any group differences, all $p \mathrm{~s}>.09$. The overall pattern of comparisons indicates that the significant interactions noted above are due to the fact that the No Shift group preferred the absolute location whereas the Shift* group preferred the relative location over the absolute location.

\section{Discussion}

The results of Experiment 2 indicate that platform placement results in a modest preference for directional responding while clearly showing that placement training does not result in a preference for navigation to the absolute location of the platform in the room. That animals appear to learn the general direction of the platform within the pool when only given platform placement training was not entirely expected. If rats effectively sample the available distal cues from the platform without active swim training then it seems reasonable to expect that the animal would learn the absolute location of the platform. Instead it appears that whatever is learned during platform placement experience is expressed as a directional response when the pool is repositioned. Because there was no active swim training the rats could not have learned to swim in a particular direction. One possibility is that rats learn the platform's location in the pool, thus, the present findings suggest an alternative interpretation of directional responding (see General Discussion). Of course, the quality of discrimination between absolute and relative locations in the Shift and No Shift groups is clearly not on par with that observed after active swim training, nonetheless, the data indicate that whatever is learned is expressed as a directional response rather than a place response. Together with the results of Experiment 1, these observations suggest that the range of situations in which directional responding occurs in the water task is rather broad. Although interesting and important, a more complete understanding of why platform placement experience supports directional responding will require additional experimentation. Pursuit of this particular question will not be trivial, and more importantly, will not further address the primary goals of the present study. In the interest of further evaluating the generality of directional responding in the water task, the remainder of the experiments reported here attempt to determine whether other methods that involve active swim training support navigation to absolute places or directional responding.

\section{Experiment 3}

Experiments 1 and 2 demonstrated that rats prefer directional responding over place navigation in situations where the platform location remained fixed throughout training. Although these observations suggest that directional responding predominates true place navigation in standard water task procedures, it is important that the potential for other commonly used water task protocols to support true place navigation over directional responding be evaluated. One such procedure is the moving platform variant of the water task (Steele \& Morris, 1999; Whishaw, 1985) in which the platform is routinely relocated during training. Although it seems reasonable to suspect that the moving and fixed platform protocols do not qualitatively differ with respect to what is learned, it is also possible that moving platform training could favor true place navigation over directional responding. Some considerations that helped establish the basic rationale for Experiment 3 included the possibilities that moving platform training may favor place navigation by (1) enhancing the formation and/or influence of a viewindependent representation of space such as a cognitive map (Nadel \& Hardt, 2004; O'Keefe \& Nadel, 1978; Tolman, 1948), (2) enhancing learning about distal cues and their relationships to precise spatial locations in the pool, or (3) otherwise reducing the influence of direction because the direction of the platform in the room and apparatus would not be reliable throughout training. Of course, the absolute location of the platform in the moving platform task is also not reliable throughout training and a clear alternative possibility is that rats will continue to display a preference for directional responding over place navigation despite the use of multiple platform locations and directions during training. If so, the results would further establish the generality of directional responding in basic water task protocols. To evaluate these predictions, rats were given hidden platform training in which the platform was moved to a novel location after every fourth trial ( 9 platform locations/ 36 trials total); After the final training trial a probe trial was conducted with the pool in the same position (No Shift group) or repositioned (Shift group) such that the final platform location (location B, see Figure 1) was in the opposite quadrant. Preference for directional responding or navigation to the absolute spatial location was evaluated as in the previous experiments.

\section{Method}

Subjects. Subjects were 16 experimentally naive rats that were randomly assigned in equal numbers $(n=8)$ to the Shift or No Shift groups.

Apparatus. The testing room (room 1) and pool were the same as those used in Experiment 1.

Design and procedure. All animals received 3 blocks of 4 hidden-platform training trials during each of 3 daily sessions (for a total of 9 trial blocks). Prior to each block the platform was relocated to a novel position. The sequence of platform locations was constrained such that (1) each location was in a different quadrant and was a different distance from the pool wall from the location that preceded it and (2) the platform was in location B during the final block (block 9) regardless of the pool position. Eight animals received training with the pool in position 1 (4 per group) and the other 8 received training with the pool in position 2 (4 per group). Following the final training trial a $30 \mathrm{~s}$ no-platform probe trial was conducted with the pool repositioned (Shift group) or with the pool in the same location as used during training (No Shift group).

\section{Results}

Hidden platform training. Because the platform was relocated at the beginning of each trial block, group comparisons (No Shift 
vs. Shift) were performed individually for each training trial rather than on averages for each trial block. There were no significant group effects for any individual training trial, all $p$ s $>.077$. Mean latencies for the final four trials (final platform location) were $22.75 \mathrm{~s}, 11.56 \mathrm{~s}, 6.23 \mathrm{~s}$, and $5.31 \mathrm{~s}$, respectively. Mean group latencies for the final training trial were $5.38 \mathrm{~s}$ and $5.25 \mathrm{~s}$ for the No Shift and Shift groups, respectively.

No-platform probe trial. Representative probe trial swim paths for rats from the No Shift and Shift groups of Experiment 3 are shown in Figure 6 and group means for each of the probe trial dependent measures are shown in Figure 7. During the probe trial, 7 of the 8 No Shift rats navigated to the absolute region first, whereas 6 of the 8 Shift rats navigated to the relative region first. One Shift rat (Rat \#6) began a trajectory directly toward the relative location and then changed course prior to entering the relative region and ultimately entered the absolute region first. Another Shift rat (Rat \#14) navigated directly to the absolute location. Separate ANOVAs were conducted for each of the four probe trial dependent measures with group (No Shift or Shift) and location (absolute vs. opposite/relative) as factors. There was a significant Location $\times$ Group interaction for latency to enter each region, $F(1,14)=11.85$; however, none of the remaining main effects for latency were significant, both $p s>.20$. There were no significant main effects or interactions for the remaining measures: number of crosses, all $p \mathrm{~s}>.21$; region proximity, all $p \mathrm{~s}>.06$; and Time in Region, all $p s>.22$.

Animals in the No Shift group navigated into the absolute region faster than the opposite region, $F(1,7)=17.92$, whereas animals in the Shift group navigated to the relative region faster than the absolute region, although this difference was not significant, $F(1$, $7)=1.62, p=.24$. In observing the performance of individual animals in the Shift group, the lack of a significant difference in latencies for the Shift group can be attributed to the performance of a single animal. Six of the Shift animals had latencies to enter the relative region ranging from $1.83-2.83 \mathrm{~s}$ and corresponding latencies to enter the absolute region ranging from $6.50-13.50 \mathrm{~s}$. One animal (Rat \#14) had a latency of $6.5 \mathrm{~s}$ to enter the relative region and a latency of $2.00 \mathrm{~s}$ to enter the absolute region, con-

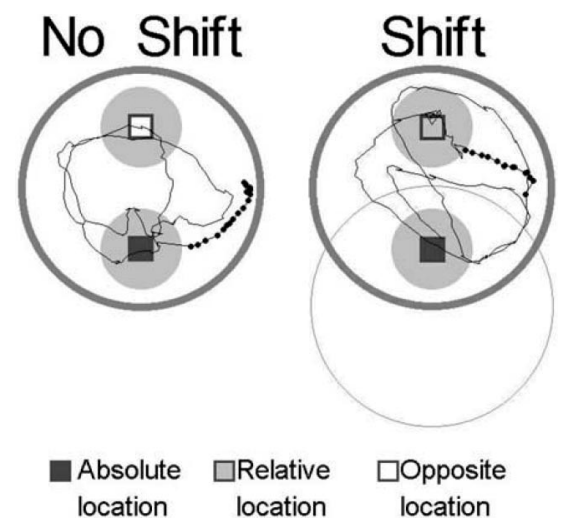

Figure 6. Representative probe trial swim paths for animals from the Shift and No Shift groups of Experiment 3. Paths were selected for animals with median latencies to enter the preferred region during the probe trial (absolute for the No Shift group and relative for the Shift group). Conventions for representing locations, critical regions, pool positions, and swim paths are the same as those described for Figure 2. sistent with a preference for the absolute region. Another Shift animal (Rat \#6) had a latency of $13.5 \mathrm{~s}$ to enter the relative region, which falls more than 2.2 standard deviations above the group mean, and did not take a direct path to either region. The inclusion of Rat \#6 increased the Shift group mean by $44 \%$. Thus, we also conducted a comparison of latencies to enter the relative and absolute location for the Shift group with Rat \#6 excluded from the analysis, which resulted in a significant location effect; relative $<$ absolute, $F(1,6)=6.19$ (mean latencies for the Shift group with Rat \#6 excluded are represented as Shift* in Figure 7).

Rats in the No Shift group navigated to the absolute region faster than rats in the Shift group regardless of whether Rat \#6 was excluded, both $p \mathrm{~s}<.006$. Shift rats navigated into the relative region faster than No Shift rats navigated into the opposite region regardless of whether Rat \#6 was included in the analyses, both $p \mathrm{~s}<.02$. A comparison of latencies to the absolute region for the No Shift group and latencies to the relative region for the Shift group failed to detect a difference regardless of whether Rat \#6 was included, both $p s>.43$. Comparisons of latency to the absolute region in the Shift group and latency to the opposite region of the No Shift group also failed to detect differences regardless of the inclusion of Rat \#6, both $p s>.18$.

\section{Discussion}

The results of Experiment 3 are consistent with a preference for directional responding over place navigation after moving platform training. Most animals in the Shift group navigated to the relative region first when the pool was repositioned, and as a group, Shift animals navigated to the relative region more quickly than they navigated to the absolute region. Animals in the No Shift group entered the absolute region first, resulting in lower latencies to enter the absolute region compared to the opposite region. With respect to measures related to persistence in searching (number of platform crosses, time in region, and average distance), neither group displayed a clear preference for either of the two critical regions. Training in the moving platform task reduces persistence and focused search at the platform location, thus, emphasis should be placed on the initial trajectory which clearly supports the conclusion that repositioning the pool resulted in a preference for navigation in the direction of the platform in the pool over navigation to the absolute location. As such, the results of Experiment 3 establish that moving platform training does not fundamentally alter the basic pattern of directional responding observed when the platform location is fixed.

\section{Experiment 4}

Experiment 4 was conducted to determine whether methods that have been useful in training navigation to absolute places in dry land mazes would also be successful in the water task. Other studies (Blodgett et al., 1949; Skinner et al., 2003; Stringer et al., 2005) have demonstrated a dissociation between direction and place in dry-land mazes by moving the maze (e.g., plus-maze or T-maze) or open field for every trial and keeping the location of reinforcement in the same absolute location in the room or in the same direction (same relative location in the apparatus). Pilot research in which the pool was repositioned between the two pool positions (shown in Figure 1) and the platform remained in the same place or direction revealed that rats will learn to navigate to 

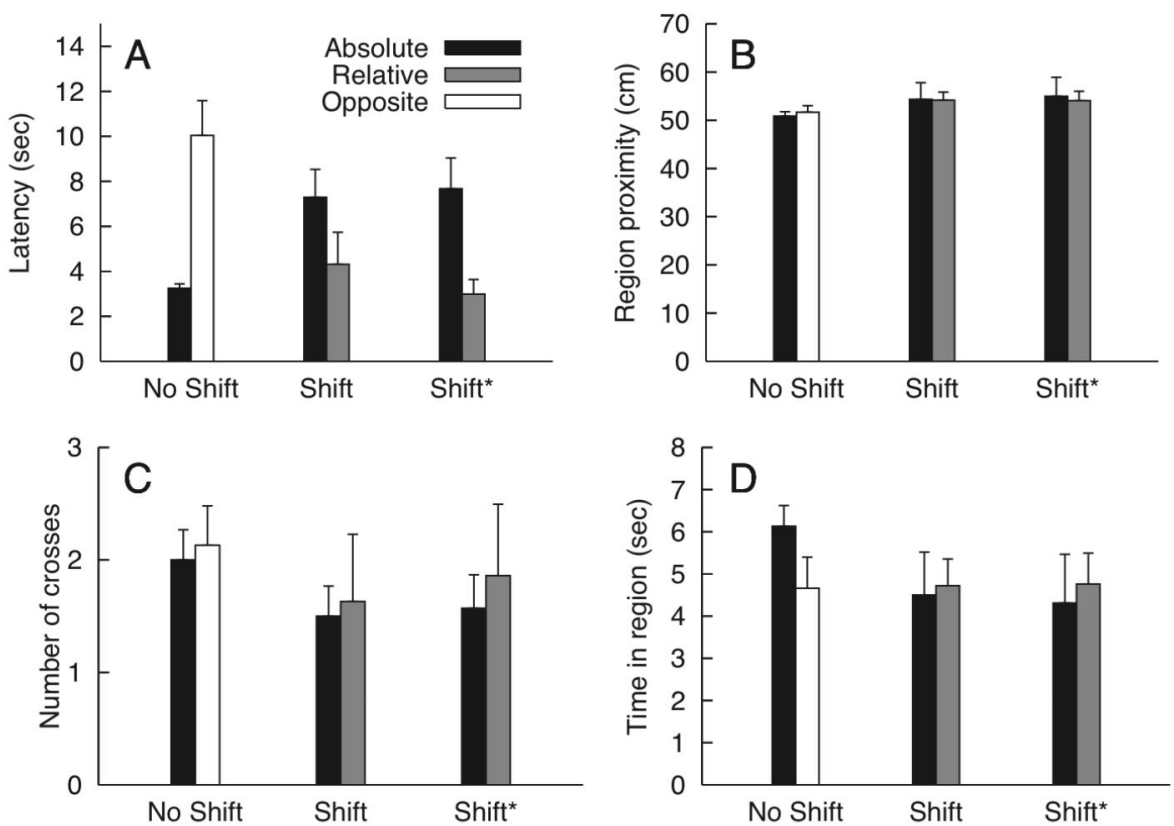

Figure 7. Probe trial dependent measures (Mean + SEM) for the No Shift and Shift groups of Experiment 3. Shift* represents mean performance for the Shift group with one outlier (Rat \#6) excluded. A: Latency to enter the $66 \mathrm{~cm}$ diameter circular region around the two locations of interest. B: Average distance from the two critical locations. C: Number of times each critical location was crossed. D: Time spent in each of the two critical circular regions.

the trained location, but will also swim back and forth on the principal axis along which the pool was moved. Thus, this particular approach did not provide compelling evidence regarding the ability of rats to learn place responses as the data could also be explained in terms of learning a generalized form of directional responding (swimming back and forth). Pilot work further highlighted the fact that apparent place navigation can reasonably be explained in terms of learning separate directional responses for each pool position; simply navigating directly to the absolute location for each pool position is not sufficient to rule out directional responding. Experiment 4 was conducted to evaluate this possibility. During training the pool was repositioned between two locations and the platform always remained in the same place in the room. At the end of training a no-platform probe trial was conducted with the pool either in one of the trained positions or in a novel position such that the relative and absolute locations of the platform were in conflict. If rats in the latter condition prefer the absolute platform location over the relative location(s) then the ability of rats to learn place responses in at least one variant of the water task would be demonstrated and the utility of the procedures for training true place navigation would be confirmed. Alternatively, if rats prefer the relative location(s) then the predominance of directional responding over place navigation would be further established and the utility of these procedures for establishing true place navigation would be questioned.

\section{Method}

Subjects. Subjects were 16 experimentally naive rats that were randomly assigned in equal numbers $(n=8)$ to the Trained group or the Novel group.
Apparatus. Due to its larger size, room 2 (described in Experiment 2) was used rather than room 1 because the pool was moved $37.5 \mathrm{~cm}$ to $75 \mathrm{~cm}$ in any of four directions for the probe trial. This allowed sufficient room to move the pool to the required locations while also ensuring that the pool was at least $30 \mathrm{~cm}$ from any wall. The results of pilot research confirmed that rats perform a directional response in room 2; Rats showed a preference for the relative location comparable to that observed in Experiment 1.

Design and procedure. All animals received 12 hiddenplatform training trials on each of three days ( 2 blocks of 6 trials per day). The pool was moved to a different position (1 or 2) and the platform was always in the same absolute location within the room (see Figure 8). Six release points (N, NE, SE, S, SW, NW; see Figure 1) were used rather than 4 as in the previous experiments. ${ }^{1}$ Each release point was used for each pool position during each daily training session. A single no-platform probe trial was conducted immediately following the final training trial. Half of the rats were assigned to the Trained condition in which the pool

\footnotetext{
${ }^{1}$ This minor procedural deviation from previous experiments was done to ensure some variability in the distance between the release point and platform location across all individual trials for each pool position. Because the pool is moved prior to each trial, the use of 6 release points, and thus three different distances between the release point and platform location, ensures that at least two different distances between release locations and the platform are used for each pool position. This change in procedure avoids the possibility that using only 4 release points ( 2 distances from the platform) as in the previous experiments could result in the use of release points that are only close to or far from the platform location for each pool position.
} 


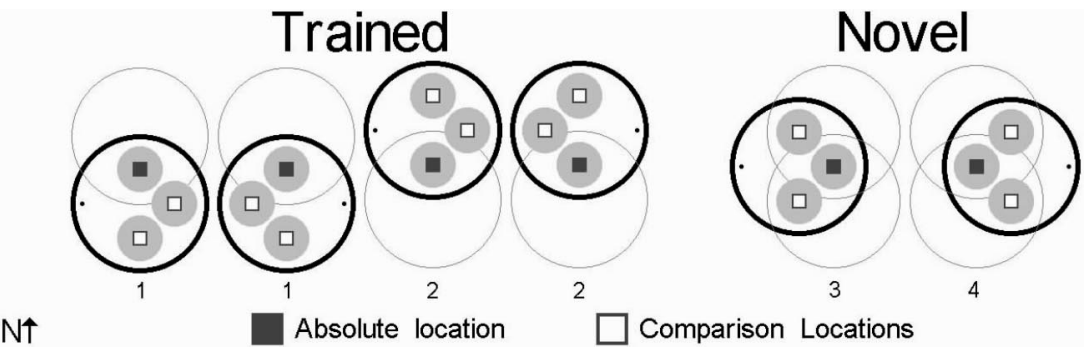

\begin{abstract}
Figure 8. Pool positions and critical analysis regions used during the probe trial of Experiment 4. The absolute (trained) location in the room and 2 comparison locations used for analysis are marked by black and white squares, respectively, and the corresponding analysis regions $(66 \mathrm{~cm}$ diameter) are marked by filled gray circles. Release points (small black circles) were always opposite one of the critical locations. The pool positions used during training are represented as thin circles and the pool positions used during the no-platform probe trial is represented as a thick circle. For both groups the pool position alternated between locations 1 (south-most position) and 2 (north-most position) during training. During the no-platform probe trial the pool was located in one of the two trained positions for the Trained group and animals were released from either the east-most or west-most locations around the perimeter of the pool. For the Novel group the pool was either in position 3 (west-most position) or 4 (east-most position), neither of which were used during training. The numbers below each configuration of training and probe trial pool positions refer to the pool position used for the no-platform probe trial. Note that the two comparison locations for the Novel group were in the relative locations where the platform was placed during training, and that one comparison region for the Trained group occupies the same relative location in the pool as the absolute location for the Novel group.
\end{abstract}

was repositioned to a location (1 or 2; see Figure 8 ) used during training and the other half were assigned to the Novel condition in which the pool was moved to a novel location for the probe ( 3 or 4; see Figure 8). For the Novel group the pool was repositioned $37.5 \mathrm{~cm}$ (half of the pool radius) in the north-south direction and $37.5 \mathrm{~cm}$ to the west (position 3 in Figure 8) or to the east (position 4 in Figure 8). This resulted in the trained platform location being in a different relative location within the pool that was the same distance from the pool wall as was used during training. For the probe trial, the pool was repositioned for all rats of both groups (i.e., moved to a location other than that used during the final training trial) and an equal number of rats from each condition received probe trials with each possible pool position ( 1 or 2 for Group Trained and 3 or 4 for Group Novel). Rats were released from either the west-most or east-most release points. Release points for rats in the Trained condition were selected such that each release point was used equally often for each pool position. Rats in Group Novel were always released from the location opposite the absolute platform location (W for pool position 3 and E for pool position 4). Probe trial dependent measures for Experiment 4 were similar to those used in the previous experiments with the exception that three critical locations were used rather than two. The absolute location was a critical location for both groups. For the Novel group the two comparison locations were centered in the quadrants adjacent to the quadrant that contained the absolute location (see Figure 8). Note that these two locations correspond to the relative locations in the pool where the platform was located during training. For the Trained group the two comparison locations were in the quadrant opposite the absolute location and in the quadrant opposite the release point (see Figure 8). Note that the latter comparison location is in the same spatial relationship to the release point as the absolute location for the Novel group.

\section{Results}

Hidden platform training. Escape latencies for each of the 6 training trial blocks were analyzed with repeated measures ANOVA with trial block (1-6) and group (Trained or Novel) as factors. There was a main effect of trial block, $F(5,70)=31.65$, which resulted from a decrease in escape latency across trial blocks, $M(S D)_{\text {Block1 }}=23.73 \mathrm{~s}(2.28) ; M(S D)_{\text {Block6 }}=6.41 \mathrm{~s}$ (0.53). The group main effect was not significant nor was there a significant Group $\times$ Trial Block interaction, both $p \mathrm{~s}>.29$.

No-platform probe trial. During the probe trial, 7 of the 8 rats in the Trained condition navigated to the absolute region first. In contrast, only 2 rats in the Novel group navigated to the absolute region first, whereas the remaining 6 rats navigated to one of the comparison regions first. All 6 of these rats showed a clear preference for the north-most comparison location. ${ }^{2}$ Representative swim paths for animals from each group are shown in Figure 9.

Group and location means for the probe trial dependent measures are shown in Figure 10. There were no significant group main effects for any of the measures, all $p$ s $>.13$. There were significant location main effects for all four dependent measures, all $p \mathrm{~s}<.005$. Overall, rats navigated into the absolute region faster

\footnotetext{
${ }^{2}$ This may indicate that the tested pool position simply generalized more readily to pool position 1 , or that some features of the environment in the north side of the room elicited greater responding directed toward that region of the pool. Note, however, that there was no evidence that animals in the Trained group only preferred this side of the pool. Animals tested with the pool in a trained position discriminated between the trained and comparison regions of the pool equally well, indicating that the direction of movement in the environment was not generally biased toward the northmost region (all of the animals were trained to navigate to the south-most region for half of the trials and all four animals tested in this condition went directly to the trained location rather than the north-most region).
} 


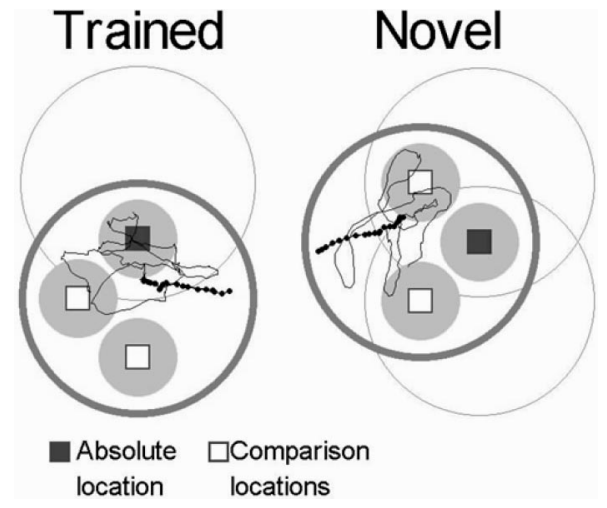

Figure 9. Representative probe trial swim paths for animals from the Trained and Novel groups of Experiment 4. Paths were selected for animals with median latencies to enter the preferred region during the probe trial (absolute location for the Trained group and one comparison location for the Novel group). Conventions for representing locations, critical regions, pool positions, and swim paths are the same as those described for Figure 2.

than at least one of the comparison regions, $F(1,14)=9.37$, spent more time in the absolute region than at least one of the comparison regions, $F(1,14)=15.63$, crossed the absolute location more frequently than at least one of the comparison regions, $F(1,14)=$ 21.41 , and navigated closer to the absolute region than at least one of the comparison regions, $F(1,14)=11.06$. There were signifi- cant Location $\times$ Group interactions for all four dependent measures, all $p s>.02$.

Separate pairwise comparisons for each location revealed that the Trained group entered the absolute region faster than both comparison locations, both $p s<.006$, spent more time in the absolute region compared to both comparison locations, both $p \mathrm{~s}<$ .007 , crossed the absolute location more frequently than both comparison locations, both $p \mathrm{~s}<.019$, and navigated closer to the absolute location than either of the two comparison locations, both $p s<.006$. In contrast, the Novel group spent more time in one of the comparison locations compared to the absolute region, $F(1$, $7)=6.16$, and navigated closer to one of the comparison locations than the absolute location, $F(1,7)=29.19$. Although the Novel group had numerically lower latencies for entry into a comparison location and crossed a comparison location more frequently than the absolute location, these comparisons were not significant, both $p$ s $>.21$.

Group comparisons for the preferred locations (absolute for the Trained group and one of the relative comparison locations for the Novel group) failed to detect significant group differences for any of the four dependent measures, all $p s>.14$. Group comparisons for the nonpreferred locations (comparison locations for the Trained group and the absolute location for the Novel group) also failed to detect significant differences, all $p \mathrm{~s}>.14$, consistent with the conclusion that animals in the Novel group treated the absolute location in much the same way that animals in the Trained group treated an arbitrarily selected location.
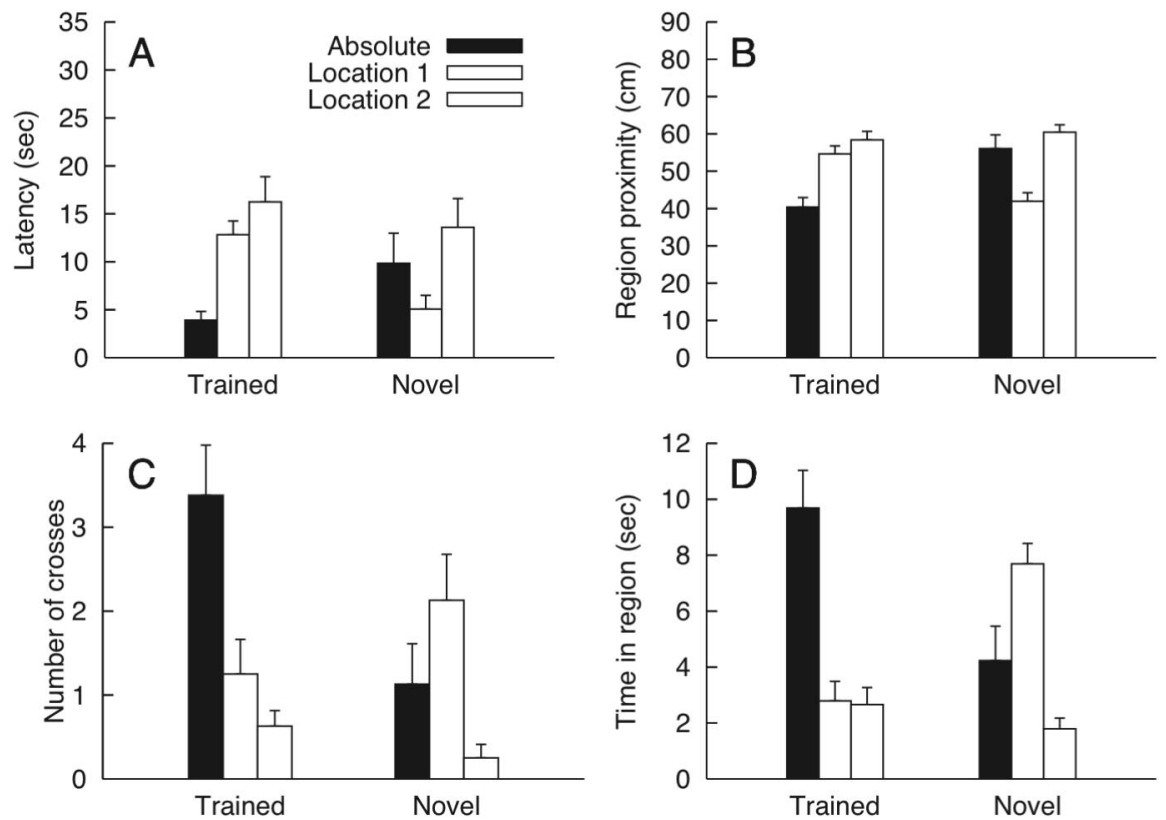

Figure 10. Probe trial dependent measures (Mean + SEM) for the Trained and Novel groups of Experiment 4. Black bars represent mean values for the absolute location of the platform in the room. White bars represent values for two comparison regions ( 1 and 2). For the Trained group comparison regions were in the quadrant adjacent to the absolute quadrant and in the quadrant opposite the absolute quadrant (see Figure 8). For the Novel condition the comparison locations were in quadrants adjacent to the absolute quadrant (see Figure 8). A: Latency to enter the $66 \mathrm{~cm}$ diameter circular region around the three locations of interest. B: Average distance from the three critical locations. C: Number of times each critical location was crossed. D: Time spent in each of the three critical circular regions. 


\section{Discussion}

The results of Experiment 4 support the conclusion that directional responding predominates true place navigate when rats are trained to navigate to a precise location regardless of the position of the apparatus in the room. Even though all rats learned to navigate to the appropriate location regardless of the pool position, rats tested with the novel pool position displayed a preference for one of relative platform locations in the pool used during training rather than the absolute location. Rats tested with a novel pool position did, however, prefer the absolute location over the other (nonpreferred) comparison location, indicating that they perhaps learned the precise location of the platform as well. The finding of major importance, however, is that moving the pool repeatedly between two locations and keeping the platform at the same place in the room neither effectively establishes true place navigation nor overcomes the influence of direction. Thus, the results of Experiment 4 further strengthen the idea that the direction is more influential than place in the water task.

\section{Experiment 5}

Based on the findings of Skinner et al. (2003) and Blodgett et al. (1949), we expected the procedures of Experiment 4 to favor true place navigation, however, a clear preference for directional responding was established. Moving the pool between two locations may not be sufficient to overcome the influence of direction because the pool is only moved along a single axis, which may account for the generalized directional responding noted in pilot work, and the absolute platform location occupies only two relative locations in the pool. As such, under these circumstances it appears that rats can learn separate directional responses (one for each pool position) more easily than they can learn a single place response. The influence of direction could perhaps be reduced if a greater number of pool positions, and therefore a greater number of axes and relative pool positions, were used during training. In Experiment 5 the pool was repeatedly moved between four locations (6 distinct axes) and the platform either remained in the same location (Place group) or in the same relative location in the pool (Direction group). A single probe trial was conducted with the pool in a novel location. These procedures should more effectively reduce the influence of direction in the Place group, and as such, should allow for a reasonable assessment of whether rats learn directional responding or place navigation at different rates.

\section{Method}

Subjects. Subjects were 16 experimentally naive rats that were randomly assigned in equal numbers $(n=8)$ to the Place group or the Direction group.

Apparatus. The testing room (room 2) and pool were the same as those used in Experiment 4.

Design and procedure. All rats received 12 hidden-platform training trials on each of three days (3 blocks of 4 trials per day). For each trial the pool was repositioned to one of four locations (see Figure 11). The pool positions and platform locations for each group during training and testing are described using $x, y$ coordinates (cm units). Using $(0,0)$ as an arbitrarily selected central point around which the pool was repositioned, the four locations at which the pool was centered during training were: location $1(0,37.5)$, location $2(37.5,0)$, location $3(0,-37.5)$, and location $4(-37.5,0)$. For the Place group the platform was always in the same absolute location in the room $(0,0)$ as shown in Figure 11 ; note that the platform occupied four separate relative locations in the pool (one for each pool position) during training. For the Direction group the platform was always in the same relative location within the pool; note that for each Direction rat the platform was located at four separate places (one per pool position) during training. Two rats were trained with the platform in the north region of the pool; the platform coordinates for pool locations $1-4$ for these two rats were $(0,75),(37.5,37.5),(0,0),(-37.5,37.5)$, respectively (see Figure 11). For the remaining Direction rats the platform was in the south $(n=2)$, east $(n=2)$, or west $(n=$ $2)$. The platform coordinates for pool locations $1-4$ were: South $[(0,0),(37.5,-37.5),(0,-75),(-37.5-37.5)]$, East $[(37.5,37.5)$, $(0,75),(37.5,-37.5),(0,0)]$, West $[(-37.5,37.5),(0,0),(-37.5,-$ $37.5),(0,-75)]$. During each training block of four trials each pool location was used once and the order of pool positions followed a pseudorandom sequence. Rats were released from one of three release points (directly opposite, to the left, or to the right of the platform) with each release point being used once for each pool position during a given daily session of 12 trials. For each trial, latency to navigate to the platform, total path length, and average distance from the platform (proximity) were measured.

After the final training trial on Day 3, a single $30 \mathrm{~s}$ probe trial was conducted with the pool in one of four novel locations (pool locations 5-8, see Figure 11) that were not used during training: location $5(26.5,26.5)$, location $6(26.5,-26.5)$, location $7(-26.5,-26.5)$, and location $8(-26.5,26.5)$. Each pool location was used for two rats from each group. The goal of the probe trial was to evaluate how well each group learned to navigate to a particular target location in the pool, therefore, the probe trial dependent measures were taken for the target location (place or direction within the pool) and a single nontarget comparison location rotated $90 \mathrm{deg}$ (around the center of the pool) to the left or right of the target location. For the Place group the target location was always at $(0,0)$ and comparison locations were $(54,0)$ or $(0,54)$ for location $5,(54,0)$ or $(0,-54)$ for location $6,(0,-54)$ or $(-54,0)$ for location 7 , and $(-54,0)$ or $(0,54)$ for location 8 . For the Direction group the target location was always the same relative location in the pool used during training; $37.5 \mathrm{~cm}$ to the north, south, east, or west of the pool coordinates given above. The nontarget location was 37.5 $\mathrm{cm}$ to the east or west of the pool center for rats trained with the platform in the north or south, and $37.5 \mathrm{~cm}$ to the north or south of the pool center for rats trained with the platform in the east or west. The release point for the probe trial was always on the opposite side of the pool and equidistant from the two critical locations. As in the previous experiments, the area covered by each location of interest was equivalent to the area of the platform, the two critical regions were $66 \mathrm{~cm}$ in diameter and centered on each critical location, and the dependent measures for each region were the same as those used in the previous experiments. 
A Training

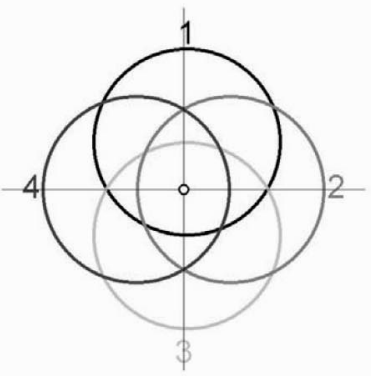

Probe

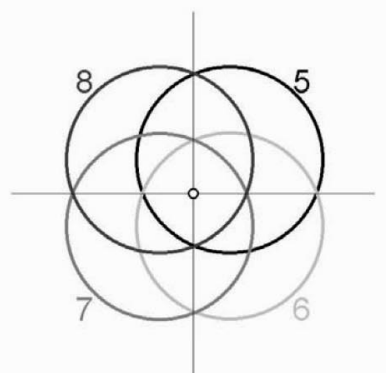

B $\quad$ Place - Training $\quad$ Place - Probe

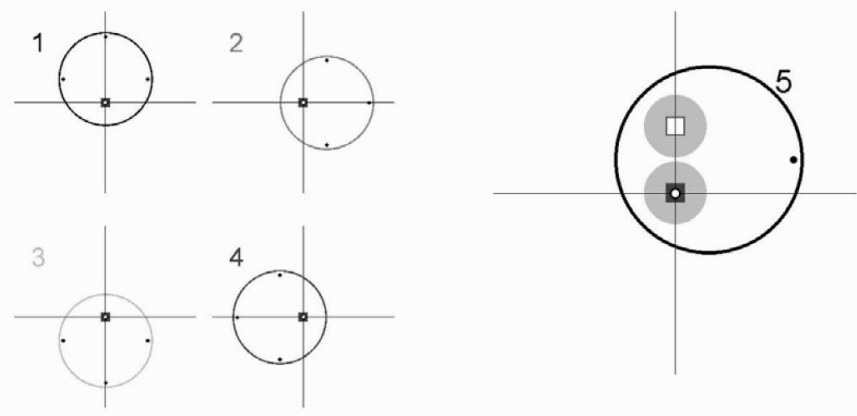

C Direction - Training Direction - Probe

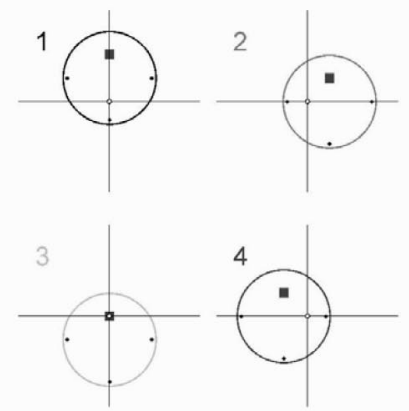

Target Location $\square$ Non-target Location

$\circ$ Origin

- Release Location(s)

Figure 11. A) Pool positions used during hidden platform training (positions 1-4) and the no-platform probe trial (positions 5-8) of Experiment 5. For all panels of this figure, the point marked by the open circle where the two lines cross was designated as the room origin. B) LEFT: The four pool positions and platform locations used during training for all rats in the Place group. RIGHT: Pool position, release point, and analysis regions used during the probe trial for one rat from the Place group. The black square and surrounding circle represent the target region and the open rectangle and surrounding circle represent the nontarget region. Small black circles mark the training and probe release points for panels B and C. C) LEFT: The four pool positions used during training for all rats in the Direction group. The platform locations shown here represent the locations used for the 2 animals trained with the platform in the north region of the pool. RIGHT: Pool position, release point, and analysis regions used during the probe trial for one rat from the Direction group (north location).

\section{Results}

Hidden platform training. Escape latencies, path length, and platform proximity were analyzed in separate repeated measures ANOVAs with trial block (1-9) and group (Place or Direction) as factors. Group means for each of the dependent measures during each trial block are shown in Figure 12. There were main effects of trial block for escape latency, $F(8,112)=29.21$, path length, $F(8,112)=29.83$, and proximity, $F(8,112)=29.87$, each of which resulted from a decrease across trial blocks. The Place group had longer mean latencies, $F(1,14)=6.73$, and mean path length, $F(1,14)=6.56$, than the Direction group. The Direction group also navigated closer to the platform on average than the Place group, however, this effect only approached significance, $F(1$, 

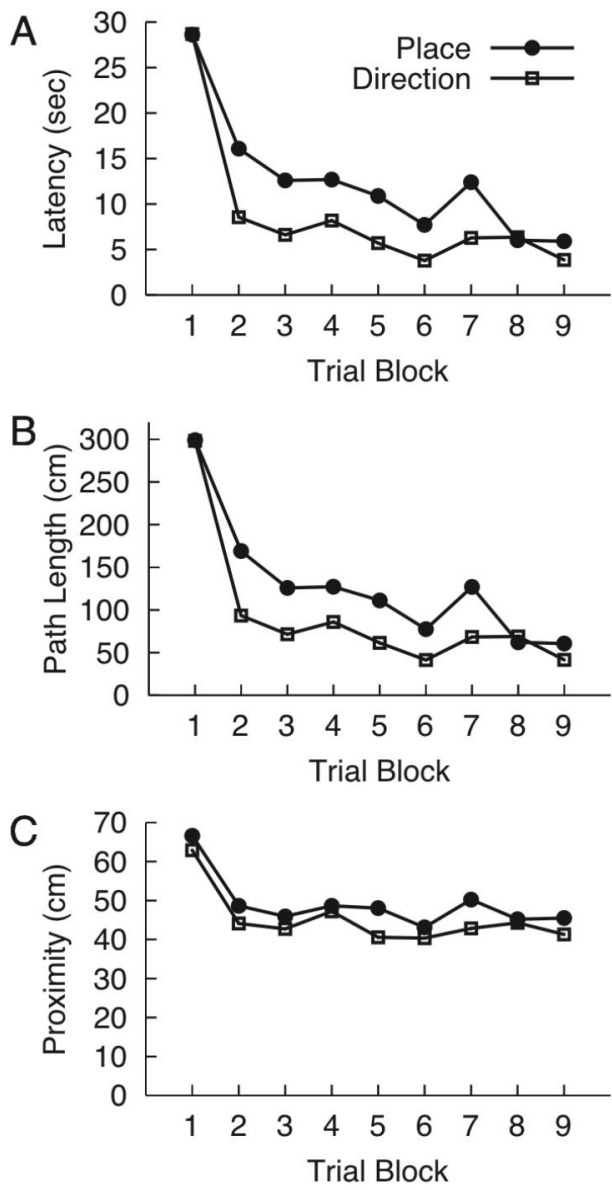

Figure 12. Mean latency (A), path length (B), and average distance (proximity) from the platform $(\mathrm{C})$ for the Place and Direction groups to navigate to the hidden platform during the 9 training trial blocks ( 4 trials each) of Experiment 5.

14) $=4.35, p=.056$. Although none of the Group $\times$ Trial Block interactions reached statistical significance (all $p s>.37$ ), it is notable that the Place and Direction groups had nearly identical means during the first trial block, but the Place group had numerically larger means for all three dependent measures during each of the subsequent blocks with the exception of block 8. Mean comparisons for each block revealed that the Place group had longer latencies than the Direction group for blocks 3, 5, 6, 7, and 9, all $p s<.041$, had longer path lengths for blocks 3, 6, 8, and 9, all $p \mathrm{~s}<.021$, and navigated further from the target location during block $5, p=.009$.

No-platform probe trial. During the probe trial, all 8 Direction rats and 6 of the 8 Place rats navigated to the target region before entering the nontarget region. Group and location (target vs. nontarget) means for the probe trial dependent measures are shown in Figure 13. The Direction group spent more time than the Place group in both regions, $F(1,14)=11.21$, however, none of the other group effects were significant (all $p \mathrm{~s}>.06$ ). There were significant location effects for all four dependent measures; latency (target $<$ nontarget), $F(1,14)=7.40$, proximity (target $<$ nontarget), $F(1,14)=24.44$, time in region (target $>$ nontarget),
$F(1,14)=18.79$, and number of crosses (target $>$ nontarget $), F(1$, $14)=7.78$. This basic pattern was observed in separate analyses for each group. For the Place group, there were significant location effects for time in region (target $>$ nontarget), $F(1,7)=15.66$, number of crosses (target $>$ nontarget), $F(1,7)=6.48$, and proximity (target $<$ nontarget), $F(1,7)=13.73$; the location effect for latency was not significant, $F(1,7)<1$. For the Direction group, there were significant location effects for time in region (target $>$ nontarget), $F(1,7)=9.53$, proximity (target $<$ nontarget), $F(1,7)=12.37$, and latency (target $<$ nontarget), $F(1,7)=$ 42.93; the location effect for number of crosses was not significant, $F(1,7)=2.77, p=.14$. None of the Location $\times$ Group interactions were significant, all $p s>.18$.

\section{Discussion}

The results of Experiment 5 establish that rats can learn to place navigate or perform a directional response in the Morris water task under conditions in which the pool is repositioned among four locations. As such, the apparent inability of rats to place navigate observed in Experiment 4 can be attributed to the use of only two pool locations. Although rats did learn to place navigate, directional responding was learned more readily, thus, the results are consistent with the previous data reported here as well as the studies of Blodgett et al. (1949) and Skinner et al. (2003). We have previously suggested that rats learn to swim in a particular direction based on distal cues at the appropriate distance from the pool wall, however, the present results along with those of Experiment 2 suggest that directional responding may involve navigating to a particular region of the pool relative to the distal cues. Thus, rather than simply providing a simple distance cue the pool wall could act as an important frame of reference. For the place groups of Experiments 4 and 5 the precise location of the platform in the room is relevant whereas the relative location of the platform in the pool is irrelevant. Using just two pool positions, and therefore two separate relative locations for the place groups, is not sufficient to establish the relevance of the critical environmental features, whereas four locations is shown here to be sufficient. Considered in this way, perhaps the methods used for the Place group of Experiment 5 effectively reduced the salience of the pool as a frame of reference while increasing the salience of the distal cue reference frame. Perhaps other manipulations that alter the salience of the relevant reference frames in this way would also support true place navigation.

\section{Experiment 6}

A common feature of the five experiments presented thus far, and the three experiments reported by Hamilton et al. (2007), was the presence of a prominent pool wall. The methods used in Experiments 1-4 failed to elicit a preference for true place navigation over directional responding, however, the results of Experiment 5 indicate that rats can learn to place navigate when the pool is moved between four locations during training and the platform remains in the same absolute location. Under these conditions the absolute location of the platform in the room is reliable whereas the relative location of the platform in the pool is not, thus, these methods may effectively reduce the salience of the apparatus. Experiment 6 directly evaluated the effects of reducing the sa- 

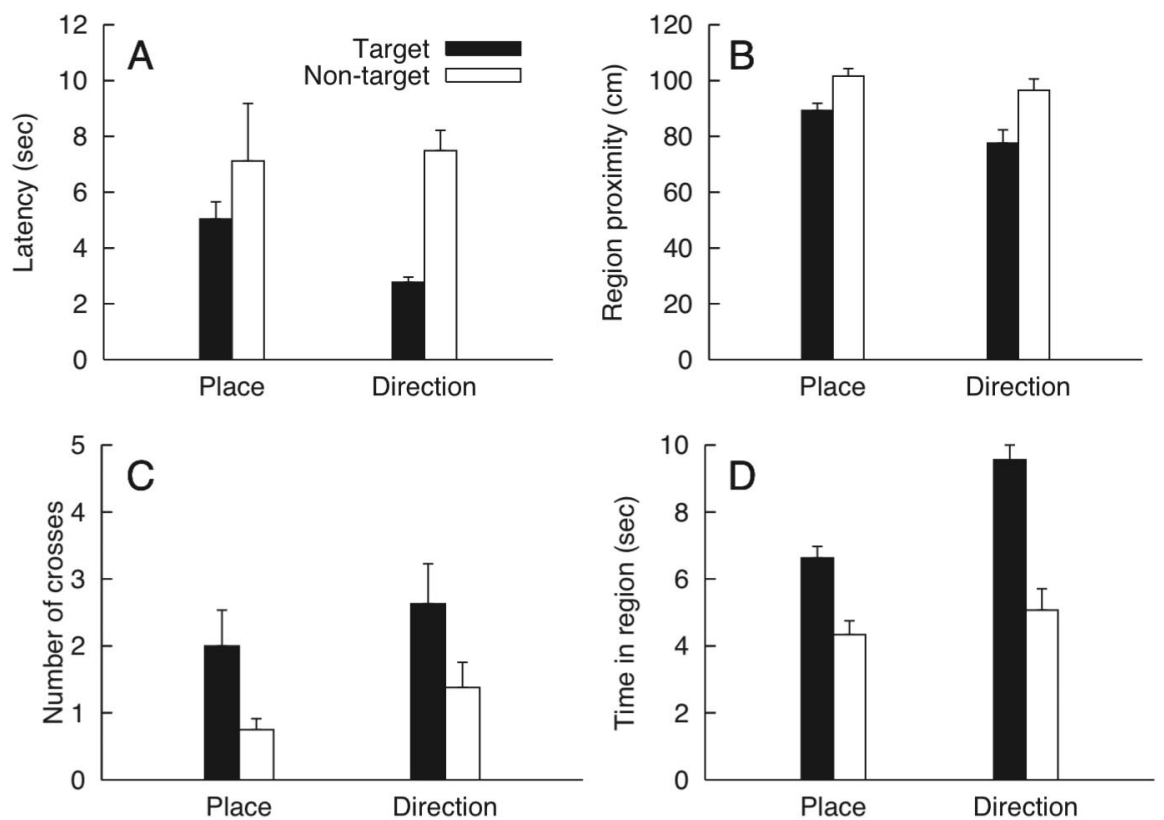

Figure 13. Probe trial dependent measures (Mean + SEM) for the Place and Direction groups of Experiment 5. Black bars represent mean values for the target location and white bars represent values for a single, nontarget comparison region located $90 \mathrm{deg}$ from the target location (see Figure 11). A: Latency to enter the $66 \mathrm{~cm}$ diameter circular region around the two locations of interest. B: Average distance from the two critical locations. C: Number of times each critical location was crossed. D: Time spent in each of the two critical circular regions.

lience of the pool wall by filling the pool with water such that it was not a prominent feature of the environment. Rats were trained with the pool and platform location fixed, after which the pool was repositioned for a no-platform probe trial as in Experiments 1-3. Preference for place navigation or directional responding were assessed as in the previous experiments. Hamilton et al. (2007) suggested that directional responding involves a trajectory based on distal cues and search at the appropriate distance from the pool wall, therefore, removal of the pool should not affect the trajectory of the swim but may influence subsequent search behavior. Alternatively, if the directional responding reflects navigating to a particular relative location in the pool then the absence of a salient apparatus reference frame may favor true place navigation based on the distal cue reference frame.

Subjects. Subjects were 16 experimentally naive rats that were randomly assigned in equal numbers $(n=8)$ to the Shift or No Shift groups.

Apparatus. The pool and room (room 1) were the same as those used Experiments 1-3. The only difference was that the pool was filled to a depth of $42 \mathrm{~cm}$, leaving $4 \mathrm{~cm}$ of the pool's inner surface visible rather than $22 \mathrm{~cm}$ as in the previous experiments. The height of the platform was adjusted accordingly so that the top of the platform was $1 \mathrm{~cm}$ below the surface of the water. Four sections of clear Plexiglas $(3 \mathrm{~mm}$ thick, $14 \mathrm{~cm}$ high, $118 \mathrm{~cm}$ in length) were arranged around the inner surface of the pool and extended $17 \mathrm{~cm}$ above the surface of the water to prevent escape from the pool. Thus, the Plexiglas sections were visible, however, in contrast to the pool wall they were transparent and did not obscure the distal visual cues.

Design and procedure. All rats were given 12 hidden platform training trials on each of two successive days. The pool was positioned at location 1 for 8 rats ( 4 Shift and 4 No Shift) and at location 2 for 8 rats ( 4 Shift and 4 No Shift). The platform was placed at location $\mathrm{B}$ for both pool positions. At the end of each of the two training sessions a probe trial was conducted either with the pool in the same position as used in training (No Shift) or with the pool repositioned to the other possible pool position (Shift). The rationale for using fewer training trials (12) prior to the probe trial than in the previous experiments and testing at the end of each day was based on the possibility that animals may navigate to the absolute platform location early in training and switch to another form of responding later in training. Although the results of Hamilton et al. (2007) established that rats perform directional responding early as well as after more extensive training, this was only established in cases where the pool wall was a prominent feature of the environment. Thus, the present experiment was designed to address the issue of whether rats navigate to the absolute platform location during the tests when the animals were given minimal training to reach asymptote.

\section{Results}

Hidden platform training. Latency data were averaged for each of the 6 training trial blocks and analyzed with repeated measures ANOVA with trial block (1-6) and group (No Shift and Shift) as factors. There was a significant main effect of trial block, $F(5,70)=28.02$, resulting from a decrease in escape latency across the trial blocks; $M(S D)_{\text {Block1 }}=30.61 \mathrm{sec}$ (10.94), $M(S D)_{\text {Block6 }}=4.84 \mathrm{sec}$ (2.36). The main effect of group was not significant, $F(1,14)<1$, as was the interaction, $F(5,70)<1$. Mean group latencies during the final trial block were $4.69 \mathrm{~s}$ (No Shift) and $5.00 \mathrm{~s}$ (Shift). 
No platform probe trial. During each of the two probe trials, seven of the eight rats in the No Shift group navigated to the absolute region first and 6 of the 8 Shift rats navigated to the absolute region first (representative swim paths for each group are shown in Figure 14). A preliminary analysis with day (1-2), location, and group as factors revealed that none of the interactions involving day were significant, all $p$ s $>.407$, thus, because the data were highly similar for both probe trials the four probe trial dependent measures for the two probe trials were averaged in order to simplify the analyses. Group means for the averaged probe trial measures are shown in Figure 15. Separate ANOVAs were conducted for each measure with group (No Shift vs. Shift) as a between-subjects factor and location (absolute vs. opposite/ relative) as a within-subject factor, where the opposite location corresponds to the relative location for the Shift group.

There were significant main effects of location for latency, absolute $<$ opposite, $F(1,14)=21.56$; number of crosses, absolute $>$ opposite, $F(1,14)=31.77$; location proximity, absolute $<$ opposite, $F(1,14)=16.23$; and time in region, absolute $>$ opposite, $F(1,14)=49.82$. Rats in the No Shift group crossed locations more frequently than Shift rats, $F(1,14)=9.52$, and spent more time in the regions of interest, $F(1,14)=16.48$, however, the group main effects for latency and region proximity were not significant, both $p s>$.14. There were significant Location $\times$ Group interactions for all four dependent measures, all $p \mathrm{~s}<.02$.

Follow-up comparisons for locations within each group revealed that No Shift animals entered the absolute region faster than the opposite region, spent more time in the absolute region, crossed the absolute location more frequently, and navigated closer to the absolute location, all $p$ s $<.049$. Rats in the Shift group entered the absolute region faster than the relative region, $F(1,7)=5.67$; and spent more time in the absolute region, $F(1,7)=5.56$; however, the location effects were not significant for number of crosses or region proximity, both $p \mathrm{~s}>.14$. Nonetheless, it should be noted that, numerically, there was a preference for the absolute location over the relative location for both measures.

Separate group comparisons for the absolute and opposite measures revealed that the No Shift animals navigated to the absolute

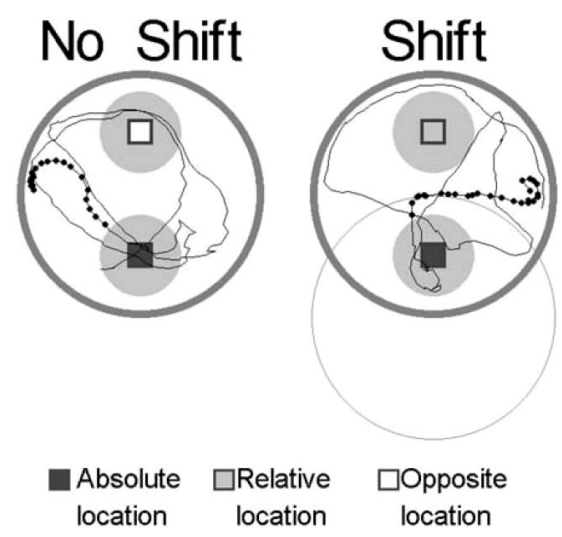

Figure 14. Representative probe trial swim paths for animals from the No Shift and Shift groups of Experiment 6. Paths were selected for animals with median latencies to enter the preferred region during the probe trial (absolute for both groups). Conventions for representing locations, critical regions, pool positions, and swim paths are the same as those described for Figure 2. region faster than Shift animals, spent more time in the absolute region, crossed the absolute location more frequently, and navigated closer to the absolute location, all $p \mathrm{~s}<.047$. Rats in the Shift group navigated to the relative region faster than No Shift animals navigated to opposite region, and Shift animals also navigated closer to the relative region than No Shift animals navigated to the opposite region, both $p$ s $<.041$. There were no group differences for time in the relative versus opposite region or number of times these locations were crossed, both $p s>.12$. Overall, the follow-up comparisons indicate that the significant interactions reported above are due to differences in the magnitude of effects for each location, but not due to a group difference in the direction of effects as has been observed in the previous experiments.

\section{Discussion}

The results of Experiment 6 are in clear contrast to the results of Experiments 1-5 reported here, as well as the results of Hamilton et al. (2007). With the salience of the pool wall reduced, the Shift group displayed a significant preference for the absolute location for several probe trial measures, although the Shift group did not distinguish between the two locations as clearly as the No Shift group. Considered along with the previous demonstrations of directional responding when the pool wall was a prominent cue, these results suggest that the pool wall and distal cues interact in the standard water task. When the pool wall is visible rats display a preference for navigation to the relative platform location unless explicitly trained to place navigate. When the pool wall is not visible, rats navigate to the precise location where the platform was located in the distal cue reference frame.

\section{General Discussion}

The results of the present experiments provide consistent and strong evidence that directional responding predominates navigation to precise spatial locations in a broad range of procedures and techniques that are typically used in the Morris water task. The breadth of situations for which directional responding was observed suggests that this form of navigation represents the primary means by which animals navigate from the release point to a hidden escape platform in the water task. Evidence that rats can learn to place navigate in the water task was observed in two experiments, however, a preference for true place navigation over directional responding was only obtained when the pool wall was virtually eliminated as a cue. Overall, the results indicate that the relative influence of direction is greater than that of place information when cues from the apparatus are prominent, whereas the influence of place is effective when control by the apparatus is reduced.

Because the distinction between directional responding and true place navigation is a critical feature of all the experiments discussed here, and is of central importance to the interpretations and conclusions presented below, it is important to establish precisely what is meant by these terms. Using the description of Olton, Becker, and Handelmann (1979), a place would be analogous to a single point, whereas direction is involved in orientation along a line. When the pool is repositioned as in the present experiments, the absolute spatial location of the platform is put into conflict with the relative location of the platform in the pool. Place navigation 

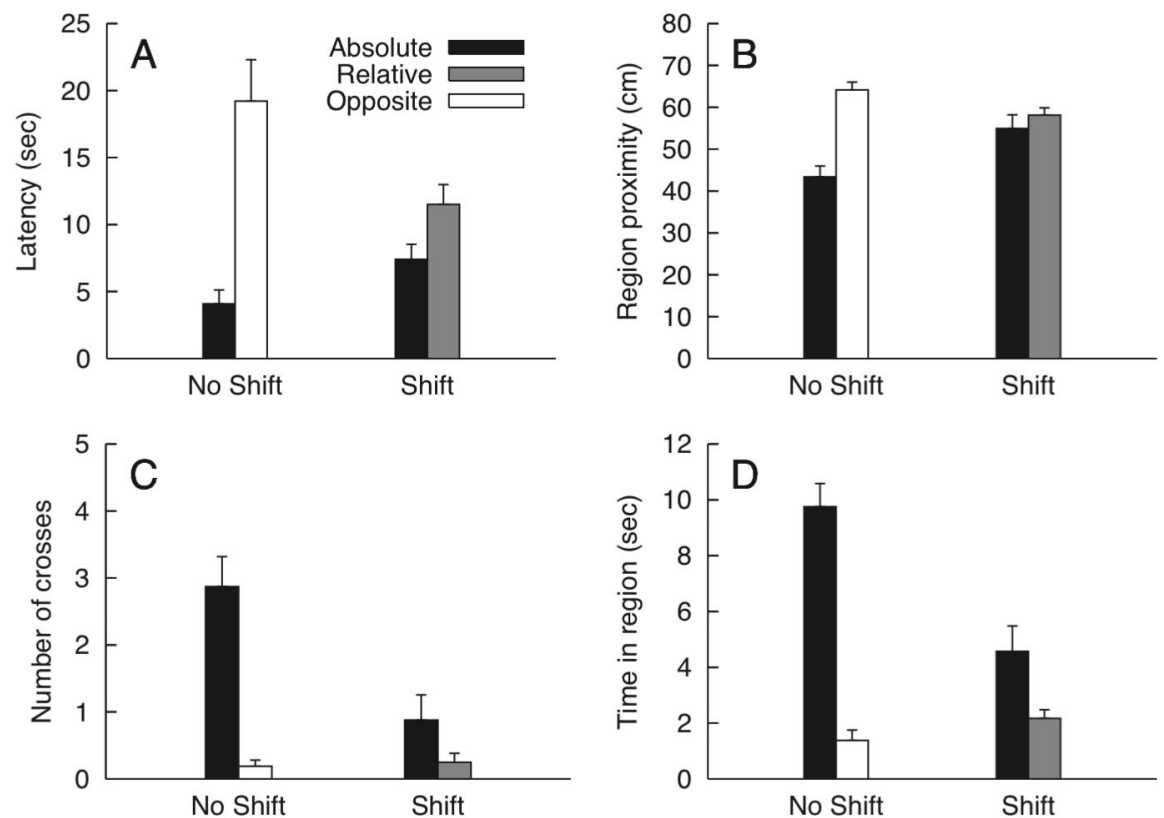

Figure 15. Probe trial dependent measures (Mean + SEM) for the No Shift and Shift groups of Experiment 6. A: Latency to enter the $66 \mathrm{~cm}$ diameter circular region around the two locations of interest. B: Average distance from the two critical locations. C: Number of times each critical location was crossed. D: Time spent in each of the two critical circular regions.

would result in navigation to the absolute location of the platform, whereas swimming in the direction of the platform in the room and pool would result in navigation to the relative location in the pool. For the purposes of the discussion that follows, the term directional responding is used to refer to this behavior. Both forms of navigation can be distinguished from simple turning responses and route learning because multiple release points (to the left or right of the platform) were used during training and novel release points were used during the critical probe trials. Although explanations of directional responding have been offered and are certainly of importance for the discussion that follows, meeting the primary goals of evaluating the range of situations in which directional responding predominates place navigation, and what, if any, procedures favor place navigation, can be reasonably achieved by simply evaluating whether evidence for directional responding or place navigation was observed without regard to a particular explanation of either behavior. Thus, we begin by summarizing the major findings with respect to this critical behavioral dissociation and commenting on the significance of the observations to current issues in the literature. After highlighting other major features of the data, we discuss the significance of the present findings for understanding spatial navigation in the water task, evaluate potential explanations of directional responding, and further distinguish directional responding from other explanations of spatial navigation in the water task.

Together with the results of Hamilton et al. (2007), the results of Experiments 1 and 2 indicate that directional responding predominates place navigation after minimal training, including platform placement training alone, as well as after more extensive training. In several dry land maze studies it has been claimed that rats shift from navigating to the place where reinforcement is located early in training to response-based navigation (e.g., turning responses) as training continues (Packard \& McGaugh, 1996; Chang \& Gold, 2003). Perhaps more generally, any form of responding that is simpler than place navigation may become the dominant form of responding with continued training, and directional responding can reasonably be considered a simpler form of responding than place navigation (Blodgett et al., 1949; Skinner et al., 2003; Stringer et al., 2005; Hamilton et al., 2007). Hamilton et al. (2007) reported a preference for directional responding with a small number of training trials (8) and we report a preference, albeit modest, for directional responding after one platform placement trial (Experiment 2). These observations score against the idea that rats place navigate early in training and later shift to directional responding. Extensive training (up to 240) trials (Experiment 1) also resulted in a clear preference for directional responding. Given that Hamilton et al. (2007) observed a preference for directional responding after 8 and 36 training trials using the same room and pool positions as used in Experiments 1 and 2 here, there is little to suggest that there is a transition from one form of navigation to another, including the possibility that animals shift from directional responding to place navigation after extensive training.

The preference for directional responding after passive platform placement training in Experiment 2 was somewhat unexpected, in part because we assumed that this type of experience would favor learning the precise spatial location of the platform. Further, active swim training is clearly a more effective means of establishing accurate swim trajectories than simply viewing cues from the platform (Chew et al., 1989; Sutherland et al., 1987), thus, we assumed that the directionality of swim trajectories based on distal cues would require active swim training. Several studies have shown that platform placement training facilitates later learning 
(Devan et al., 2002; Keith \& McVety, 1988; Sutherland \& Linggard, 1982), however, whether this facilitation reflects true place learning has been questioned (Chew et al., 1989). Given that no active swim training was provided it seems rather difficult to conclude that anything about swimming in a particular direction could have been learned. Nonetheless, whatever was learned during placement must have been sufficient to favor a directional response. One possibility is that the modest preference for directional responding reflects learning where the platform is located in the pool (i.e., the relative location). Rats could have also learned the distance between the pool wall and the platform, which is one component of the movement vector described by Hamilton et al. (2007), but this would not account for the directionality of the swim during the probe trial. Although the results of Experiment 2 indicate only a modest preference for directional responding after platform placement, that no evidence for place navigation was observed represents an important feature of the data. Future studies are needed to elucidate precisely how rats learn to perform a directional response in the absence of active swim training.

The first two experiments demonstrated that directional responding predominates place navigation so long as the platform remains in the same location during training. We reasoned that regularly moving the platform location might support true place navigation because the use of multiple goal locations could result in more extensive learning about the distal environment or otherwise enhance learning about the precise spatial location of the platform. Further, given that the rats would swim to and possibly explore more of the environment these procedures could support the formation of a cognitive map (O'Keefe \& Nadel, 1978). The results of Experiment 3 provide modest evidence that rats perform a directional response after training in a moving platform variant of the water task (Steele \& Morris, 1999; Whishaw, 1985), while clearly demonstrating that rats do not prefer to place navigate. Although the rationale of Experiment 3 could be questioned, there is substantive value in the fact that the training procedures could have favored place navigation but did not, therefore, this outcome further extends the generality of the preference for directional responding over place navigation in the water task.

Given that rats do not appear to place navigate in several typical variants of the water task, an extreme possibility is that rats are simply not capable of true place navigation, or that the influence of direction is so strong that evidence for place navigation cannot be observed in this task. Studies using dry land mazes (Blodgett et al., 1949; Skinner et al., 2003; Stringer et al., 2005) have demonstrated that rats can learn to place navigate when the apparatus is repositioned during training and the site of reinforcement in the room is held constant. These studies have also shown that rats generally learn directional responses more readily than place responding, thus, the results of Experiments 1-3 and the findings from dry maze studies are largely consistent despite the differential emphasis on training versus testing manipulations. Experiments 4 and 5 evaluated whether rats could learn to take direct trajectories to a platform in a fixed location when the pool was repositioned from trial to trial. When the pool was repositioned between two locations during training rats either swam back and forth on the principal axis the pool was moved along (pilot observations), or navigated to one of the relative platform locations used during training (Experiment 4) rather than learning to place navigate. Place navigation was successfully trained when the pool was repositioned among four different locations (Experiment 5), therefore, it appears that moving the pool along multiple axes is necessary to overcome the influence of direction. Consistent with the dry land maze studies, the results of Experiment 5 also firmly establish that rats learn directional responses more readily than place navigation in the water task. Given that evidence for true place navigation required an extreme departure from typical methods, the results question whether rats can learn to place navigate in the standard water task where the methods would appear to be insufficient for overcoming the rather significant influence of direction.

One discrepancy between the results of some dry land maze studies and those of Experiment 5 warrants discussion. Skinner et al. (2003) found that rats learned a place response just as well as a directional response provided that the spatial locations of the release points for the various apparatus positions could be easily discriminated. This was achieved by taking paths of different lengths from the holding cage to each of the release points in addition to increasing the physical distance between release points. Consistent with these observations, Horne, Martin, Harley, and Skinner (2007) demonstrated that sensitivity to multiple release locations in the plus maze is required for true place navigation. There were no situations in the present where rats learned to place navigate as well as they learned to perform a directional response (see below for clarification regarding Experiment 6). Multiple distinct release points around the perimeter of the pool were used in all of our experiments and since the holding cages were in a fixed location the length and direction of the paths taken by the experimenter to the release point were necessarily of different lengths. It is difficult to imagine that the rats could not discriminate between release points that were typically separated by up to $1.5 \mathrm{~m}$ ( $2.25 \mathrm{~m}$ in Experiment 5). The apparent discrepancy between the dry land and water task results likely reflect basic differences in the two procedures and apparatus that were employed. Future studies should attempt to determine whether the spatial relationships among release points influence whether rats learn directional responses or true place navigation in the water task. On the basis of the present findings we would predict that the influence of direction would be greater than that of place regardless of minor parametric changes in the basic methods, however, the findings of Horne et al. (2007) clearly suggest that sufficiently increasing the size of the pool could favor true place navigation.

When the pool wall was virtually eliminated as a cue in Experiment 6 rats not only learned to place navigate but showed a preference for place navigation over directional responding. Given that rats could have learned to swim in a particular direction based on the distal cues but did not, it appears that the pool wall influences whether distal cues provide directional or place cues. One possibility is that the reduction in the pool wall increased the distinctiveness of the release points and, therefore, favored place navigation (Skinner et al., 2003; Horne et al., 2007). It is also possible that reducing the pool wall's salience resulted in a corresponding increase in the salience of the distal cues. Restle (1957) suggested that the salience of the distal visual environment would influence whether rats perform place navigation or rely on some other form of responding such as simple motor responses. Although Restle's analysis may not apply to the distinction between directional responding and place navigation since both depend on distal cues, it is nonetheless important to consider whether the pool 
wall obscured the distal cues to the degree that they were unable to control place navigation; a related, albeit extreme, possibility is that not much of the distal environment was visible to the rat when the pool was half full. Of course, if it is accepted that accurate performance in the standard water task depends upon the distal visual cues, then control by the available visual stimuli should be apparent in the rather direct nature of the trajectories taken to the platform (as was observed in all the experiments reported here). Increasing the height of the pool wall necessarily decreases the amount of visual information provided by the distal cues, however, as described in the General Method section, the majority of the putative controlling stimuli were placed high up on the walls such that they were easily visible whether the pool was full or half full. To evaluate whether the pool wall had a detrimental effect on learning we compared escape latencies for rats trained with a full pool (Experiment 6) or a prominent pool wall (Experiment 1). Rats trained with a pool wall actually learned to escape faster than rats trained with a full pool, although both reached comparable levels of performance after 16-24 trials. ${ }^{3}$ Thus, if anything the presence of the pool wall facilitated learning despite the reduction in visual information. ${ }^{4}$ Further, the distal cues used in these studies were capable of controlling place navigation even when the pool wall was visible (see Experiment 5), however, directional responding was the preferred form of navigation in all situations in which the pool wall was present. Finally, although the possibility that the pool wall reduces control by the distal cues is certainly worth considering in future studies, in our opinion this possibility does little to detract from the primary importance of the current data with respect to the question of what is learned in the water task. That is, the presence of a prominent pool wall, and its associated effects on the visibility of distal room cues, is a characteristic feature of the water task as it is typically employed. We presume that the preference for directional responding over place navigation reported here will generalize to most situations in which the standard procedures and apparatus are employed.

A final noteworthy feature of the results that warrants some discussion concerns the magnitude of the preference for the relative platform location when the pool is repositioned compared to the preference for the absolute location when the pool is not repositioned. Although we have routinely characterized the performance of the Shift groups as treating the relative location much the same way as rats in the No Shift groups treat the absolute location, inspection of the probe trial means suggests that the Shift group showed less of a preference for the relative location. To evaluate this general impression we performed an analysis including data for all Shift and No Shift animals from Experiments $1-3^{5}$, treating the relative and absolute locations as target locations, respectively. Shift rats spent less time in the target region and crossed the target region less frequently than No Shift rats, however, there were not detectable differences for latency to enter the target region or proximity to the target region. ${ }^{6}$ Thus, the group differences with respect to target location are modest and it seems reasonable to attribute them to a generalization decrement. Shift rats experienced a rather large change in the environment that was not present for the No Shift animals; thus, some decrement in performance should be expected. Considering that the pool was moved a distance equal to the radius of the pool, it is perhaps surprising that there was not more of a quantitative decrement in performance. The fact that Shift rats treated the relative location much as the No Shift group treated the absolute despite the rather large change in the environment supports the conclusion that directional responding can account for performance in the standard water task when the pool remains stationary.

Hamilton et al. (2007) proposed that directional responding in the water task involves a movement vector in which distal cues control the directionality of the trajectory, while navigation to and search at a precise location within the pool is controlled by distance information from the pool wall. Pearce, Roberts, and Good (1998) utilized a variant of the water task in which rats navigated to an escape platform that was always a fixed distance and direction from a conspicuous proximal cue. This form of vector-based responding bears some similarity to the directional responding described here, however, in the present experiments there were no proximal cues other than the pool itself. Pearce et al. (1998) found that rats with hippocampal damage could perform vector-based responding with a single proximal cue but could not solve the standard water task. This neurobehavioral dissociation can be taken to indicate that rats do not solve the standard water task using vector-based navigation with respect to the distal cues and pool wall, thus, it seems reasonable to conclude that vectorbased responding and the movement vector described by Hamilton et al. (2007) with respect to the pool wall and distal cues reflect different processes. The possibility that rats respond to the apparatus as a frame of reference in which particular locations within the apparatus are disambiguated by the distal cues represents a clear alternative to the movement vector hypothesis of Hamilton et al. (2007). Stated another way, rats could learn to navigate to the region of the pool closest (or furthest) to a particular region of the distal cue environment. Such an explanation more readily accounts for the data showing that platform placement results in directional responding and the data of Experiment 5 showing that rats can easily be trained to navigate to a relative location when the pool is moved along multiple axes in the room. The notion that rats take a trajectory based solely on the distal cues is difficult to reconcile with the observations of Experiment 2 since there was no active swim training in which taking such a trajectory could be learned. Accurate navigation in the Direction group of Experiment 5 could

\footnotetext{
${ }^{3}$ Comparisons of mean escape latencies for 6 trial blocks (4 trials each) were conducted for rats trained with the full pool (Experiment 6) and rats trained with the pool half full (Experiment 1). Rats trained with the full pool had significantly longer escape latencies than rats trained with the pool half full during blocks 2,3 , and 5 (all $p$ s $<.02$, all other $p \mathrm{~s}>.08$ )

${ }^{4}$ It is of some importance that the two conditions compared here differed only in the presence of the pool wall, therefore, directional responding cannot be attributed to the presence of general directional cues (e.g. sounds, smells) as these were present in both situations and should have resulted in directional responding regardless of whether the pool was visible or not.

5 The analyses were limited to these experiments because in the other experiments the pool was either not shifted for the probe trial, the concept of an absolute location did not apply, or there was a preference for the absolute location in the Shift group

${ }^{6}$ No Shift rats $(n=32)$ spent more time in the absolute region than Shift rats $(n=32)$ spent in the relative region, and crossed the absolute location more frequently than Shift rats crossed the relative location (both $p \mathrm{~s}<$ .03). Latency to enter the target region and proximity, however, did not differ (both $p s>.12$ ).
} 
not be achieved by simply taking a single trajectory in a particular direction in the room because the precise trajectory would differ for each pool position and release point. Further, the observation that rats performed a place response rather than a directional response when the pool was full is inconsistent with the idea that the distal cues determine the trajectory of the swim independently of the apparatus, but is consistent with the idea that rats respond to the apparatus reference frame with respect to the distal reference frame. The distinction can perhaps be expressed more clearly by considering the importance of the pool wall for determining the directionality of the trajectory according to each hypothesis. If rats set a trajectory based on distal cues and then search at the appropriate distance from the pool wall as suggested by Hamilton et al.(2007) then the pool wall is of little relevance to the initial direction of the swim. In contrast, if animals respond to locations in the apparatus that are disambiguated by distal cues, then the apparatus is a critical determinant of the direction of the swim. The latter view can be taken to imply that when the pool is present as a frame of reference, the distal cues will serve to disambiguate locations within the apparatus, but when the pool is not a salient reference frame, the distal cues will disambiguate locations within the distal reference frame alone, and, therefore, place navigation is supported. Viewed in this way, the evidence for directional responding with a prominent apparatus cue, and place navigation when the salience of the pool is reduced, are collectively consistent with the operation of distinct reference frames (Gibson, 2001; Sutherland \& Hamilton, 2004) or separate, nonglobal maps (Cheng, 1986; Gallistel, 1990) in the standard water task.

It is important to note that the explanations offered above are not mutually exclusive, nor are they exhaustive, as other processes may contribute to the observed behavior. What can be stated with certainty is that these explanations are in clear contrast to explanations based on the notion that animals navigate to precise spatial locations on the basis of a mental representation of space, such as a cognitive map (Nadel \& Hardt, 2004; O'Keefe \& Nadel, 1978; Tolman, 1948;), or by attempting to match perceptual memory of the available visual stimuli from the goal site (Wilkie \& Palfrey, 1987). Directional responding is inconsistent with the operation of perceptual matching, however, perceptual matching could account for performance when the salience of the pool wall is reduced. In fairness to Wilkie and Palfrey (1987), their perceptual matching model assumes that the apparatus is not a critical source of control. Although a preference for directional responding does not refute mapping theory, the idea that rats place navigate in the water task based on a global cognitive map or any unified, conjunctive representation is difficult to reconcile with the consistent preference for directional responding reported here. For example, consider the possibility that a global representation including the pool and distal cues is established during training, and when the pool is shifted this representation is for lack of a better term, morphed. A problem with this view is that the clear preference for the relative location when the pool is shifted must indicate that the relative location is more like the absolute location than the absolute location itself. In our opinion, a more parsimonious explanation is that the pool and distal cues function to control behavior interactively as described above.

Two broad implications of the present findings are that (1) the typical Morris water task does not measure true place navigation, but rather measures directional responding and (2) that scientific questions regarding the processes involved in true place navigation in the water task should be addressed using methods that support true place navigation. These would include reducing control by the pool wall, although there are likely to be other preparations that support true place navigation. It is important to note that directional responding was observed in two distinct distal environments in the present study, suggesting that the precise makeup of the distal cues was not a critical determinant of whether directional responding or place navigation occurred. We do, however, acknowledge the possibility that particular configurations of the distal cues could differentially support directional responding or place navigation. Given that testing in cue-controlled environments (e.g., Devan et al., 2002; Prados \& Trobalon, 1998; Rodrigo, Chamizo, McLaren, \& Mackintosh, 1997) represents an important segment of the literature on spatial navigation, it will be important to establish whether specific cue configurations that support true place navigation can be identified. Should it turn out that directional responding predominates place navigation in an even broader range of environments and procedures than is described here, there are clear implications for precisely what behaviors are in need of explanation as well as how scientific questions regarding navigation in the water task should be framed. If directional responding generally predominates place navigation, then appealing to theoretical processes involved in true place navigation to explain Morris water task performance would not be necessary. In general, future research should focus on further characterizing the processes involved in directional responding in order to better understand the behavioral processes involved in navigation from one location to another (Mackintosh, 2002; Shettleworth, 1998). Methods such as those used here will be useful in attempts to understand these basic processes, and more generally, may serve an important role in further elucidating the constituent processes involved in spatial navigation.

\section{References}

Blodgett, H. C., McCutchan, K., \& Mathews, R. (1949). Spatial learning in the T-maze: The influence of direction, turn, and food location. Journal of Experimental Psychology, 39, 800-809.

Chang, Q., \& Gold, P. E. (2003). Intra-hippocampal lidocaine injections impair acquisition of a place task and facilitate acquisition of a response task in rats. Behavioural Brain Research, 144, 19-24.

Cheng, K. (1986). A purely geometric module in the rat's spatial representation. Cognition, 23, 149-178.

Chew, G. L., Sutherland, R. J., \& Whishaw, I. Q. (1989). Latent learning does not produce instantaneous transfer of place navigation: A rejoinder to Keith and McVety. Psychobiology, 17, 207-209.

Deutsch, J. A. (1960). The structural basis of behavior. Chicago: University of Chicago Press.

Devan, B. D., Petri, H. L., Mishkin, M., Stouffer, E. M., Bowker, J. L., Yin, P-B., et al. (2002). A room with a view and a polarizing cue: Individual differences in the stimulus control of place navigation and passive latent learning in the water maze. Neurobiology of Learning and Memory, 78, 79-99.

Gallagher, M., Burwell, R., \& Burchinal, M. (1993). Severity of spatiallearning impairment in aging-development of a learning index for performance in the Morris water maze. Behavioral Neuroscience, 107, $618-626$.

Gallistel, C. R. (1990). The organization of learning. Cambridge, MA: MIT Press.

Gibson, B. M. (2001). Cognitive maps not used by humans (homo sapiens) 
during a dynamic navigational task. Journal of Comparative Psychology, 115, 397-402.

Hamilton, D. A., Akers, K. G., Weisend, M. P., \& Sutherland, R. J. (2007). How do room and apparatus cues control navigation in the Morris water task? Evidence for distinct contributions to a movement vector. Journal of Experimental Psychology: Animal Behavior Processes, 33, 100-114.

Horne, M. R., Martin, G. M., Harley, C. W., \& Skinner, D. M. (2007). Where am I? Distal cue use requires sensitivity to start location change in the rat. Journal of Experimental Psychology: Animal Behavior Processes, 33, 92-99.

Hull, C. L. (1934a). The concept of the habit-family hierarchy and maze learning: Part I. Psychological Review, 41, 33-54.

Hull, C. L. (1934b). The concept of the habit-family hierarchy and maze learning: Part II. Psychological Review, 41, 134-152.

Hull, C. L. (1943). Principles of behavior: An introduction to behavior theory. New York: Appleton-Century-Crofts.

Keith, J. R., \& McVety, K. M. (1988). Latent place learning in a novel environment and the influences of prior training in rats. Psychobiology, $16,146-151$.

Mackintosh, N. J. (2002). Do not ask whether they have a cognitive map, but how they find their way about. Psicologia, 23, 165-185.

Morris, R. G. M. (1981). Spatial localisation does not require the presence of local cues. Learning and Motivation, 12, 239-260.

Morris, R. G. M. (1984). Developments of a water-maze procedure for studying spatial-learning in the rat. Journal of Neuroscience Methods, 11, 47-60.

Nadel, L., \& Hardt, O. (2004). The spatial brain. Neuropsychology, 18, 473-476.

O'Keefe, J., \& Nadel, L. (1978). The hippocampus as a cognitive map. Oxford, England: Clarendon Press.

Olton, D. S., Becker, J. T., \& Handelmann, G. E. (1979). Hippocampus, space, and memory. Behavioral and Brain Sciences, 2, 313-322.

Packard, M. G., \& McGaugh, J. L. (1996). Inactivation of hippocampus or caudate nucleus with lidocaine differentially affects expression of place and response learning. Neurobiology of Learning and Memory, 65, 65-72.

Pearce, J. M., Roberts, A. D. L., \& Good, M. (1998). Hippocampal lesions disrupt navigation based on cognitive maps but not heading vectors. Nature, 396, 75-77.

Pearce, J. M., Roberts, A. D. L., Redhead, E. S., \& Prados, J. (2000). The influence of passive preexposure on escape from a Morris pool. Journal of Experimental Psychology: Animal Behavior Processes, 26, 186-195.

Prados, J., \& Trobalon, J. B. (1998). Locating an invisible goal in a water maze requires at least two landmarks. Psychobiology, 26, 42-48.

Restle, F. (1957). Discrimination of cues in mazes: A resolution of the 'place-vs.-response' question. Psychological Review, 64, 217-228.
Rodrigo, T., Chamizo, V. D., McLaren, I. P. L., \& Mackintosh, N. J. (1997). Blocking in the spatial domain. Journal of Experimental Psychology: Animal Behavior Processes, 23, 110-118.

Shettleworth, S. J. (1998). Cognition, evolution, and behavior. New York: Oxford University Press.

Skinner, D. M., Etchegary, C. M., Ekert-Maret, E. C., Baker, C. J., Harley, C. W., Evans, J. H., et al. (2003). An analysis of response, direction, and place learning in an open field and $\mathrm{T}$ maze. Journal of Experimental Psychology: Animal Behavior Processes, 29, 3-13.

Steele, R. J., \& Morris, R. G. M. (1999). Delay-dependent impairment of a matching-to-place task with chronic and intrahippocampal infusion of the NMDA-antagonist D-AP5. Hippocampus, 9, 118-136.

Stringer, K. G., Martin, G. M., \& Skinner, D. A. (2005). The effects of hippocampal lesions on response, direction, and place learning in rats. Behavioral Neuroscience, 119, 946-952.

Sutherland, R. J., Chew, G. L., Baker, J. C., \& Linggard, R. C. (1987). Some limitations on the use of distal cues in place navigation by rats. Psychobiology, 15, 48-57.

Sutherland, R. J., \& Dyck, R. H. (1984). Place navigation by rats in a swimming pool. Canadian Journal of Psychology, 38, 322-347.

Sutherland, R. J., \& Hamilton, D. A. (2004). Rodent spatial navigation: At the crossroads of cognition and movement. Neuroscience Biobehavioral Reviews, 28, 687-697.

Sutherland, R. J., \& Linggard, R. (1982). Being there: A novel demonstration of latent spatial-learning in the rat. Behavioral and Neural Biology, 36, 103-107.

Tolman, E. C. (1948). Cognitive maps in rats and men. Psychological Review, 55, 189-208.

Tolman, E. C., Ritchie, B. F., \& Kalish, D. (1946). Studies in spatial learning. II. Place learning versus response learning. Journal of Experimental Psychology, 3, 221-229.

Weisend, M. P., Klein, R. L., Hoesing, J. M., Astur, R. S., Koerner, A., McDonald, R. J., et al. (1995). Morris water task: Which cues define locations? Society for Neuroscience Abstracts, 21, 1939-1939.

Whishaw, I. Q. (1985). Formation of a place learning-set by the rat: A new paradigm for neurobehavioral studies. Physiology and Behavior, 35, 139-143.

Wilkie, D. M., \& Palfrey, R. (1987). A computer simulation model of rats' place navigation in the Morris water maze. Behavior Research Methods Instruments and Computers, 19, 400-403.

Received April 27, 2007

Revision received September 11, 2007 Accepted September 11, 2007 